\title{
ON THE $p$-PSEUDOHARMONIC MAP HEAT FLOW
}

\author{
${ }^{*}$ SHU-CHENG CHANG ${ }^{1},{ }^{\sharp}$ YUXIN DONG ${ }^{2}$, AND ${ }^{\dagger}$ YINGBO HAN $^{3}$
}

\begin{abstract}
In this paper, we consider the heat flow for $p$-pseudoharmonic maps from a closed Sasakian manifold $\left(M^{2 n+1}, J, \theta\right)$ into a compact Riemannian manifold $\left(N^{m}, g_{i j}\right)$. We prove global existence and asymptotic convergence of the solution for the $p$-pseudoharmonic map heat flow, provided that the sectional curvature of the target manifold $N$ is nonpositive. Moreover, without the curvature assumption on the target manifold, we obtain global existence and asymptotic convergence of the $p$-pseudoharmonic map heat flow as well when its initial $p$-energy is sufficiently small.
\end{abstract}

\section{INTRODUCTION}

In the seminal paper of J. Eells and J. H. Sampson ([ES]), they proved the existence theorem of harmonic maps between compact Riemannian manifolds via the harmonic map heat flow when the target manifold with nonpositive sectional curvature. In our previous papers ([CC1], CC2]), we considered the following pseudoharmonic map heat flow from a closed pseudohermitian manifold $\left(M^{2 n+1}, J, \theta\right)$ into a compact Riemannian manifold $\left(N^{m}, g_{i j}\right)$ on $M \times[0, T):$

$$
\left\{\begin{array}{l}
\frac{\partial \varphi^{k}}{\partial t}=\Delta_{b} \varphi^{k}+2 h^{\alpha \bar{\beta}} \widetilde{\Gamma}_{i j}^{k} \varphi_{\alpha}^{i} \varphi_{\bar{\beta}}^{j}, \quad k=1, \cdots, m, \\
\varphi(\omega, 0)=u_{0}(\omega), \quad u_{0} \in C^{\infty}(M ; N),
\end{array}\right.
$$

1991 Mathematics Subject Classification. Primary 32V05, 32V20; Secondary 53C56.

Key words and phrases. p-pseudoharmonic map; p-pseudoharmonic map heat flow; Morse-type harnack inequality; pseudohermitian manifold; Sasakian manifold; $p$-sublaplacian.

*Research supported in part by the NSC of Taiwan.

${ }^{\sharp}$ Research supported in part by the NSFC No. 11271071.

${ }^{\dagger}$ Research supported in part by the NSFC No. 11201400, Nanhu Scholars P. for Young Scholars of XYNU. 
for $\varphi \in C^{\infty}(M \times[0, T) ; N)$. Here $\Delta_{b}$ is the sub-Laplace operator and $\widetilde{\Gamma}_{i j}^{k}$ are the Christoffel symbols of $N$. Then we proved the pseudoharmonic map heat flow (1.1) admits a unique, smooth solution $\varphi \in C^{\infty}(M \times[0, \infty) ; N)$ with subconverges to a pseudoharmonic maps $\varphi_{\infty} \in C^{\infty}(M, N)$ as $t \rightarrow \infty$, provided that $M$ is Sasakian (i.e. vanishing pseudohermitian torsion) and the sectional curvature $K^{N}$ is nonpositive. This served as the CR analogue to Eells-Sampson's Theorem ([ES]) for the harmonic map heat flow. Secondly, without the curvature assumption on the target manifold ([CS], [CD]), we showed that there exists $\varepsilon>0$ depending on $n, M, N$ and $\left\|\nabla_{b} u_{0}\right\|_{L^{\infty}(M)}$ such that for any initial data $u_{0} \in C^{\infty}(M ; N)$, if the energy is small enough

$$
E\left(u_{0}\right)=\int_{M}\left|\nabla_{b} u_{0}\right|^{2} d \mu \leq \varepsilon,
$$

then the solution $\varphi$ of (1.1) exists for all $t>0$. Moreover, as $t \rightarrow \infty, \varphi(t)$ converges to a constant map. Here $\nabla_{b}$ is the subgradient on the holomorphic subbundle $T_{1,0} M \oplus T_{0,1} M$.

In this paper, we extend the above results to the $p$-pseudoharmonic map heat flow (1.4) on $M \times[0, T)$. Let $\left(M^{2 n+1}, J, \theta\right)$ be a closed pseudohermitian manifold and $\left(N^{m}, g_{i j}\right)$ be a compact Riemannian manifold. At each point $x \in M$, we may take a local coordinate chart $U_{x} \subset M$ of $x$ and a local coordinate chart $V_{\varphi(x)} \subset N$ of $\varphi(x)$ such that $\varphi\left(U_{x}\right) \subset V_{\varphi(x)}$. For a $C^{1}$-map $\varphi: M \rightarrow N$, we define the energy density $e(\varphi)$ of $\varphi$ at the point $\omega \in U_{x}$ by

$$
e(\varphi)(\omega)=\frac{1}{2} h^{\alpha \bar{\beta}}(\omega) g_{i j}(\varphi(\omega)) \varphi_{\alpha}^{i} \varphi_{\bar{\beta}}^{j}
$$

Here $h_{\alpha \bar{\beta}}$ is the Levi metric on $\left(M^{2 n+1}, J, \theta\right)$. It can be checked that the energy density is intrinsically defined, i.e., independent of the choice of local coordinates. Its $p$-energy $E_{p}(\varphi)$ of $\varphi$ is defined by

$$
E_{p}(\varphi)=\frac{1}{p} \int_{M} e(\varphi)^{\frac{p}{2}} d \mu=\frac{1}{p} \int_{M}\left|\nabla_{b} \varphi\right|^{p} d \mu, \quad p>1
$$

where $d \mu=\theta \wedge(d \theta)^{n}$ is the volume element on $M$. The $p$-pseudoharmonic map is the critical point of (1.2) which is the solution of the Euler-Lagrange equation associated to its 
p-energy $E_{p}(\varphi)$

$$
\triangle_{b, p} \varphi^{k}+2\left|\nabla_{b} \varphi\right|^{p-2} \widetilde{\Gamma}_{i j}^{k} \varphi_{\alpha}^{i} \varphi_{\bar{\alpha}}^{j}=0, \quad k=1, \ldots, m
$$

where $\Gamma_{i j}^{k}$ are the Christoffel symbols of $\left(N^{m}, g_{i j}\right)$ and $\triangle_{b, p}$ is the $p$-sublaplacian

$$
\triangle_{b, p} \varphi^{k}=\operatorname{div}_{b}\left(\left|\nabla_{b} \varphi\right|^{p-2} \nabla_{b} \varphi^{k}\right)
$$

For $p=2, \triangle_{b, 2}$ is the usual sublaplacian. It is singular for $p \neq 2$ at points where $\nabla_{b} \varphi=0$. Let $S^{1, p}(M, N)$ be the Folland-Stein space (see next section for definition). We call a map $\varphi \in S^{1, p}(M, N)$ is a weakly pseudoharmonic map if it is a weak solution of (1.3). In general it is far from understood about the regularity of the weak $p$-pseudoharmonic map ([F], [FS, Theorem 21.1.], [JL], [HS], [DT], [XZ]).

In this paper, we consider the associated $p$-pseudoharmonic map heat flow on $M \times[0, T)$ :

$$
\left\{\begin{array}{l}
\frac{\partial \varphi^{k}}{\partial t}=\operatorname{div}_{b}\left(\left|\nabla_{b} \varphi\right|^{p-2} \nabla_{b} \varphi^{k}\right)+2\left|\nabla_{b} \varphi\right|^{p-2} h^{\alpha \bar{\beta}} \tilde{\Gamma}_{i j}^{k} \varphi_{\alpha}^{i} \varphi_{\bar{\beta}}^{j}, \quad k=1, \cdots, m \\
\varphi(\omega, 0)=u_{0}(\omega),
\end{array}\right.
$$

where $u_{0}: M \rightarrow N$ is the initial data which to be of class $C^{2, \alpha}$ for $0<\alpha<1$. We will follow methods of [CC1], [FR1] and [FR2] to study the global weak solutions to the $p$-pseudoharmonic map heat flow (1.4) from a closed Sasakian $\left(M^{2 n+1}, J, \theta\right)$ to a compact Riemannian manifold $\left(N, g_{i j}\right)$. In fact, we first consider the following regularized problem of (1.4) for $0<\delta<1$,

$$
\left\{\begin{array}{l}
\frac{\partial \varphi^{k}}{\partial t}=\operatorname{div}_{b}\left(\left[\left|\nabla_{b} \varphi\right|^{2}+\delta\right]^{\frac{p-2}{2}} \nabla_{b} \varphi^{k}\right)+2\left[\left|\nabla_{b} \varphi\right|^{2}+\delta\right]^{\frac{p-2}{2}} h^{\alpha \bar{\beta}} \tilde{\Gamma}_{i j}^{k} \varphi_{\alpha}^{i} \varphi_{\bar{\beta}}^{j}, \quad k=1, \cdots, m \\
\varphi(\omega, 0)=u_{0}(\omega)
\end{array}\right.
$$

on $M \times\left[0, T_{\delta}\right)$ for the regularized $p$-energy $E_{p, \delta}$ :

$$
E_{p, \delta}(\varphi)=\frac{1}{p} \int_{M}\left(\left|\nabla_{b} \varphi\right|^{2}+\delta\right)^{\frac{p}{2}} d \mu
$$

with the regularized energy density $e_{\delta}(\varphi):=\left|\nabla_{b} \varphi\right|^{2}+\delta$.

The main difficulty comes from the CR Bochner formula (3.2) with a mixed term $\left\langle J \nabla_{b} \varphi, \nabla_{b} \varphi_{0}\right\rangle_{L_{\theta}}$ involving the covariant derivative of $\varphi$ in the direction of the characteristic vector field $\mathbf{T}$, 
which has no analogue in the Riemannian case. However, by adding an T-energy density $e_{0}(\varphi)$, we are able to overcome such a difficulty and conclude that the $p$-pseudoharmonic map heat flow has a global smooth solution from a closed Sasakian manifold $\left(M^{2 n+1}, J, \theta\right)$ into a compact Riemannian manifold $\left(N^{m}, g_{i j}\right)$. More precisely, with the same spirit as in [CC1], instead of the original energy density $e_{\delta}(\varphi)$, we estimate the total energy density

$$
\widehat{e_{\delta}}(\varphi)=e_{\delta}(\varphi)+\varepsilon e_{0}(\varphi)
$$

by adding an $\mathbf{T}$-energy density

$$
e_{0}(\varphi)=g_{i j} \varphi_{0}^{i} \varphi_{0}^{j}
$$

for some positive constant $\varepsilon$ which to be determined later. We first are able to derive the Moser type Harnack inequality (Lemma 3.3 and [CC1, Theorem 1.1.]) for the total (regularized) energy density $\widehat{e}(\varphi)$ if $M$ is Sasakian. Secondly, based on [CC1], [FR1] and [FR2], we show the energy density of the regularized $p$-pseudoharmonic map heat flow (1.5) is uniformly bounded as following :

Theorem 1.1. Let $\left(M^{2 n+1}, J, \theta\right)$ be a closed Sasakian manifold and $(N, g)$ be a compact Riemannian manifold. Let $u_{0} \in C^{2, \alpha}(M, N), 0<\alpha<1$ and $\left\|\nabla_{b} u_{0}\right\|_{L^{\infty}(M)} \leq K$.

(i) There exists $\varepsilon_{0}>0$ depending on $K, M, N$ such that if

$$
E_{p}\left(u_{0}\right)=\frac{1}{p} \int_{M}\left|\nabla_{b} u_{0}\right|^{p} d \mu \leq \varepsilon_{0}
$$

then the solution $\varphi_{\delta}$ of (1.5) satisfies

$$
\left\|\nabla_{b} \varphi_{\delta}\right\|_{L^{\infty}\left(\left[0, T^{\prime}\right) \times M\right)} \leq C \quad \text { and } \quad\left\|\mathbf{T} \varphi_{\delta}\right\|_{L^{\infty}\left(\left[0, T^{\prime}\right) \times M\right)} \leq C
$$

where $C$ is a constant depending on $K, M$ and $N$.

(ii) In addition, if the sectional curvature of $\left(N, g_{i j}\right)$ is nonpositive

$$
K^{N} \leq 0
$$

then (1.7) holds without the smallness assumption (1.6). 
(iii) The energy inequality will be

$$
\int_{0}^{t} \int_{M}\left|\partial_{s} \varphi_{\delta}\right|^{2}(x, s) d \mu d s+E_{p, \delta}\left(\varphi_{\delta}(\cdot, t)\right)=E_{p, \delta}\left(u_{0}\right) \leq E_{p, 1}\left(u_{0}\right), \quad \forall t \in\left[0, T_{\delta}\right) .
$$

Based on (1.7), (1.8) and the CR divergence theorem and CR Green's identity as in [CCW, Lemma 3.2. and Corollary 3.1.], it follows from [DF], [D], [Ch], [HS] and [LSU] that we can prove the global existence and asymptotic convergence of the $p$-pseudoharmonic map heat flow, provided that the sectional curvature of the target manifold $N$ is nonpositive.

Theorem 1.2. Let $\left(M^{2 n+1}, J, \theta\right)$ be a closed Sasakian manifold and $(N, g)$ be a compact Riemannian manifold. If the sectional curvature is nonpositive

$$
K^{N} \leq 0
$$

and $u_{0} \in C^{2, \alpha}(M, N), 0<\alpha<1$, then there is a unique global weak solution $\varphi$ of (1.4) with $\partial_{t} \varphi \in L^{2}(M \times[0, \infty))$ and $\varphi, \nabla_{b} \varphi \in C^{\beta}(M \times[0, \infty), N)$, where $0<\beta<1$. Moreover, there exists a sequence $t_{k} \rightarrow \infty$ such that $\varphi\left(t_{k}\right)$ converges in $C^{1, \beta^{\prime}}(M, N)$ for all $\beta^{\prime}<\beta$, to a weakly p-pseudoharmonic map $\varphi_{\infty} \in C^{1, \beta}(M, N)$ satisfying

$$
E_{p}\left(\varphi_{\infty}\right) \leq E_{p}\left(u_{0}\right)
$$

Moreover, without the curvature assumption on the target manifold but with small initial p-energy, we have

Theorem 1.3. Let $\left(M^{2 n+1}, J, \theta\right)$ be a closed Sasakian manifold and $(N, g)$ be a compact Riemannian manifold. Let $u_{0} \in C^{2, \alpha}(M, N), 0<\alpha<1$ and $\left\|\nabla_{b} u_{0}\right\|_{L^{\infty}(M)} \leq K$. There exists $\varepsilon_{0}>0$ depending only on $K, M, N$ such that if

$$
E_{p}\left(u_{0}\right)=\frac{1}{p} \int_{M}\left|\nabla_{b} u_{0}\right|^{p} d \mu \leq \varepsilon_{0}
$$

then there is a unique global weak solution $\varphi$ of (1.4) with $\partial_{t} \varphi \in L^{2}(M \times[0, \infty))$ and $\varphi, \nabla_{b} \varphi \in$ $C^{\beta}(M \times[0, \infty), N)$, where $0<\beta<1$. Moreover, there exists a sequence $t_{k} \rightarrow \infty$ such that 
$\varphi\left(t_{k}\right)$ converges in $C^{1, \beta^{\prime}}(M, N)$ for all $\beta^{\prime}<\beta$, to a weakly p-pseudoharmonic map $\varphi_{\infty} \in$ $C^{1, \beta}(M, N)$ satisfying

$$
E_{p}\left(\varphi_{\infty}\right) \leq E_{p}\left(u_{0}\right)
$$

Moreover, there exists $\overline{\varepsilon_{0}}>0$ depending only on $K, M, N$ and $p$ such that if in addition $E_{p}\left(u_{0}\right) \leq \overline{\varepsilon_{0}}$, then $\varphi_{\infty}$ is a constant map.

Remark 1.1. 1. One may also compare (1.4) to the well-known $p$-harmonic map heat flow from a closed Riemannian manifold $\left(M, h_{i j}\right)$ to compact Riemannian manifold $\left(N^{m}, g_{i j}\right)$ :

$$
\left\{\begin{array}{l}
\frac{\partial u}{\partial t}=\triangle_{p}^{M} u+|\nabla u|^{p-2} A(u)(\nabla u, \nabla u), \\
u(\omega, 0)=u_{0}(\omega)
\end{array}\right.
$$

where $A$ is the second fundamental form of $N$ in $R^{m+k}$. In the papers of [FR1] and [FR2], A. Fardoun and R. Regabaoui proved that if the sectional curvature $K^{N}$ is nonpositive, then the heat flow (1.9) has a unique global weak solution $u$. Moreover, $u(t)$ converges to a $p$ harmonic map $u_{\infty}$. Without the curvature assumption on the target manifold, they showed that if $u_{0} \in C^{2, \alpha}(M, N), 0<\alpha<1$ with the small $p$-energy for $p \leq \operatorname{dim} M$, then there exists a unique global weak solution $u$ of (1.9). Moreover, as $t \rightarrow \infty, u(t)$ converges to a constant map. For the further study of global weak solutions to the $p$-harmonic map heat flow (1.9), we refer to [CHH], [H1], [H2], etc for some details. We also refer to [HL for the gradient estimate of the minimizing $p$-harmonic map.

2. Since $(2 n+2)$ is homogeneous dimnesion of a pseudohermitian manifold $M^{2 n+1}$, then when $p>2 n+2$, we do not need the smallness assumption in Theorem 1.3. By following the same steps, the result of Theorem 1.3 holds for any smooth initial data. Thus we may assume that $p \leq 2 n+2$ in the proof.

3. In the paper of [CDRY, Remark 5.3.], the second named author proved that $f: M \rightarrow$ $N$ is harmonic if and only if $f$ is pseudoharmonic whenever $M$ is Sasakian and $N$ is a Riemannian manifold with nonpositive curvature. It is interesting to know whether it is true for $p$-harmonic maps and $p$-pseudoharmonic maps. 
Acknowledgments. The third named author would like to express his thanks to Taida Institute for Mathematical Sciences (TIMS), National Taiwan University. Part of the project was done during his visit to TIMS.

\section{Preliminaries}

We first introduce some basic materials on pseudohermitian $(2 n+1)$-manifolds ([L]). Let $(M, \xi)$ be a $(2 n+1)$-dimensional, orientable, contact manifold with contact structure $\xi$, $\operatorname{dim}_{R} \xi=2 n$. A CR structure compatible with $\xi$ is an endomorphism $J: \xi \rightarrow \xi$ such that $J^{2}=-i d$. We also assume that $J$ satisfies the following integrability condition: if $X$ and $Y$ are in $\xi$, then so are $[J X, Y]+[X, J Y]$ and $J([J X, Y]+[X, J Y])=[J X, J Y]-[X, Y]$. A CR structure $J$ can extend to $C \otimes \xi$, and decomposes $C \otimes \xi$ into the direct sum of $T_{1,0} M$ and $T_{0,1} M$, which are eigenspaces of $J$ with respect to eigenvalues $i$ and $-i$, respectively. A manifold $M$ with a $\mathrm{CR}$ structure is called a CR manifold. A pseudohermitian structure compatible with $\xi$ is a $\mathrm{CR}$ structure $J$ with $\xi$ together with a choice of contact form $\theta$. Such a choice determines a unique real vector field $T$ transverse to $\xi$, which is called the characteristic vector field of $\theta$, such that $\theta(T)=1$ and $L_{T} \theta=0$. Let $\left\{T, Z_{\alpha}, Z_{\bar{\alpha}}\right\}$ be a frame of $T M \otimes C$, where $Z_{\alpha}$ is any local frame of $T_{1,0} M, Z_{\bar{\alpha}} \in T_{0,1} M$, and $\mathbf{T}$ is the charecteristic vector field. Then, $\left\{\theta, \theta^{\alpha}, \theta^{\bar{\theta}}\right\}$, which is the coframe dual to $\left\{T, Z_{\alpha}, Z_{\bar{\alpha}}\right\}$, satisfies $d \theta=i h_{\alpha \bar{\beta}} \theta^{\alpha} \wedge \theta^{\bar{\beta}}$, for some positive definite hermitian matrix of functions $\left(h_{\alpha \bar{\beta}}\right)$. Locally, one can choose $Z_{\alpha}$ appropriately so that $h_{\alpha \bar{\beta}}=\delta_{\alpha \beta}$ to simplify tensorial calculation.

The Levi form $\langle,\rangle_{L_{\theta}}$ is the Hermitian form on $T_{1,0} M$ defined by

$$
\langle Z, W\rangle_{L_{\theta}}=-i\langle d \theta, Z \wedge \bar{W}\rangle
$$

We can extend $\langle,\rangle_{L_{\theta}}$ to $T_{0,1} M$ by defining $\langle\bar{Z}, \bar{W}\rangle_{L_{\theta}}=\langle, Z, \bar{W}\rangle_{L_{\theta}}$ for all $Z, W \in T_{1,0} M$. The Levi form induces naturally a Hermitian form on the dual bundle of $T_{1,0} M$, denoted by $\langle,\rangle_{L_{\theta}^{*}}$, and hence on all the induced tensor bundles. Integrating the Hermitian form over $M$ with respect to the volume form $d \mu=\theta \wedge(d \theta)^{n}$, we get an inner product on the space of sections 
of each tensor bundle. We denote the inner product by notation $\langle$,$\rangle . For example,$

$$
\langle u, v\rangle=\int_{M} u \bar{v} d \mu
$$

for functions $u$ and $v$.

The pseudohermitian connection of $(J, \theta)$ is the connection $\nabla$ on $T M \otimes C$ (and extended to tensors) given in terms of local frames $Z_{\alpha} \in T_{1,0} M$ by $\nabla Z=\omega_{\alpha}^{\beta} \otimes Z_{\beta}, \nabla Z_{\bar{\alpha}}=\omega_{\bar{\alpha}}^{\bar{\beta}} \otimes Z_{\bar{\beta}}$, $\nabla T=0$, where $\omega_{\alpha}^{\beta}$ are the 1-forms uniquely determined by the following equations:

$$
\begin{aligned}
d \theta^{\beta} & =\theta^{\alpha} \wedge \omega_{\alpha}^{\beta}+\theta \wedge \tau^{\beta} \\
0 & =\tau_{\alpha} \wedge \theta^{\alpha} \\
0 & =\omega_{\alpha}^{\beta}+\omega_{\bar{\beta}}^{\bar{\alpha}}
\end{aligned}
$$

We can write (by Cartan lemma) $\tau_{\alpha}=A_{\alpha \gamma} \theta^{\gamma}$, with $A_{\alpha \gamma}=A_{\gamma \alpha}$ the pseudohermitian torsion of $(M, J, \theta)$. The curvature of this Tanaka-Webster connection, expressed in terms of the coframe $\left\{\theta=\theta^{0}=\theta, \theta^{\alpha}, \theta^{\bar{\alpha}}\right\}$, is

$$
\begin{aligned}
& \Pi_{\beta}^{\alpha}=\overline{\Pi_{\bar{\beta}}^{\bar{\alpha}}}=d \omega_{\beta}^{\alpha}-\omega_{\beta}^{\gamma} \wedge \omega_{\gamma}^{\alpha}, \\
& \Pi_{0}^{\alpha}=\Pi_{\alpha}^{0}=\Pi_{0}^{\bar{\beta}}=\Pi_{\bar{\beta}}^{0}=\Pi_{0}^{0}=0 .
\end{aligned}
$$

Webster showed that $\Pi_{\beta}^{\alpha}$ can be written

$$
\Pi_{\beta}^{\alpha}=R_{\beta \rho \bar{\sigma}}^{\alpha} \theta^{\rho} \wedge \theta^{\bar{\sigma}}+W_{\beta \rho}^{\alpha} \theta^{\sigma} \wedge \theta-W_{\beta \bar{\rho}}^{\alpha} \theta^{\bar{\rho}} \wedge \theta+i \theta_{\beta} \wedge \tau^{\alpha}-i \tau_{\beta \wedge \theta^{\alpha}}
$$

where the coefficients satisfy

$$
R_{\beta \bar{\alpha} \rho \bar{\sigma}}=\overline{R_{\alpha \bar{\beta} \bar{\sigma} \rho}}=R_{\bar{\alpha} \beta \bar{\sigma} \rho}=R_{\rho \bar{\alpha} \beta \bar{\sigma}}, \quad W_{\beta \bar{\alpha} \gamma}=A_{\beta \gamma, \bar{\alpha}}
$$

We will denote components of covariant derivatives with indices preceded by comma; thus write $A_{\alpha \beta ; \gamma}$. The indices $\{0, \alpha, \bar{\alpha}\}$ indicate derivatives with respect to $\left\{T, Z_{\alpha}, Z_{\bar{\alpha}}\right\}$. For derivatives of a scalar function, we will often omit the comma, for instance, $\varphi_{\alpha}=Z_{\alpha} \varphi$, $\varphi_{\alpha \bar{\beta}}=Z_{\bar{\beta}} Z_{\alpha} \varphi-\omega_{\alpha}^{\gamma}\left(Z_{\bar{\beta}}\right) Z_{\gamma} \varphi, \varphi_{0}=\mathbf{T} \varphi$ for a smooth function $\varphi$. 
For a smooth real function $\varphi$, the subgradient $\nabla_{b}$ is defined by $\nabla_{b} \varphi \in \xi$ and $\left\langle Z, \nabla_{b} \varphi\right\rangle_{L_{\theta}}=$ $d \varphi(Z)$ for all vector fields $Z$ tangent to contact plane. Locally $\nabla_{b} \varphi=\sum_{\alpha}\left(\varphi_{\bar{\alpha}} Z_{\alpha}+\varphi_{\alpha} Z_{\bar{\alpha}}\right)$. We can use the connection to define the subhessian as the complex linear map

$$
\left(\nabla^{H}\right)^{2} \varphi: T_{1,0} \oplus T_{0,1} \rightarrow T_{1,0} \oplus T_{0,1}
$$

by

$$
\left(\nabla^{H}\right)^{2} \varphi(Z)=\nabla_{Z} \nabla_{b} \varphi
$$

We also define the subdivergence operator $\operatorname{div}_{b}(\cdot)$ by

$$
\operatorname{div}_{b}(W)=W^{\beta}{ }_{\beta}+W^{\bar{\beta}}, \bar{\beta}
$$

for all vector fields $W=W^{\beta} Z_{\beta}+W^{\bar{\beta}} Z_{\bar{\beta}}$. In particular

$$
\left|\nabla_{b} \varphi\right|^{2}=2 \varphi_{\alpha} \varphi_{\bar{\alpha}}, \quad\left|\nabla_{b}^{2} \varphi\right|^{2}=2\left(\varphi_{\alpha \beta} \varphi_{\bar{\alpha} \bar{\beta}}+\varphi_{\alpha \bar{\beta}} \varphi_{\bar{\alpha} \beta}\right)
$$

and

$$
\triangle_{b} \varphi=\operatorname{div}_{b}\left(\nabla_{b} \varphi\right)=\left(\varphi_{\alpha \bar{\alpha}}+\varphi_{\bar{\alpha} \alpha}\right) .
$$

We also recall below what the Folland-Stein space $S^{k, p}$ is. Let $D$ denote a differential operator acting on functions. We say $D$ has weight $m$, denoted $w(D)=m$, if $m$ is the smallest integer such that $D$ can be locally expressed as a polynomial of degree $m$ in vector fields tangent to the contact bundle $\xi$. We define the Folland-Stein space $S^{k, p}$ of functions on $M$ by

$$
S^{k, p}=\left\{f \in L^{p}: D f \in L^{p} \text { whenever } w(D) \leq k\right\} .
$$

We define the $L^{p}$ norm of $\nabla_{b} f, \nabla_{b}^{2} f, \ldots$ to be $\left(\int\left|\nabla_{b} f\right|^{p} \theta \wedge(d \theta)^{n}\right)^{1 / p},\left(\int\left|\nabla_{b}^{2} f\right|^{p} \theta \wedge(d \theta)^{n}\right)^{1 / p}$, ..., respectively, as usual. So it is natural to define the $S^{k, p}$ norm of $f \in S^{k, p}$ as follows:

$$
\|f\|_{S^{k, p}} \equiv\left(\sum_{0 \leq j \leq k}\left\|\nabla_{b}^{j} f\right\|_{L^{p}}^{p}\right)^{1 / p}
$$


The function space $S^{k, p}$ with the above norm is a Banach space for $k \geq 0,1<p<\infty$. There are also embedding theorems of Sobolev type. The reader can make reference to [F] and [FS] for more details of these spaces.

In this paper, we embed $N$ isometrically into the Euclidean space $\mathbb{R}^{l}$ with $l$ large enough and then $S^{k, p}=S^{k, p}\left(M, \mathbb{R}^{l}\right)$. Let $\pi: \mathbb{R}^{l} \rightarrow N$ be a smooth projection. Define $S^{k, p}(M, N)$ by $\pi\left(S^{k, p}\right)$ (similarly, $\operatorname{Lip}(M, N):=\pi\left(\operatorname{Lip}\left(M, \mathbb{R}^{l}\right)\right)$ and so do other spaces of maps from $M$ to $N)$. From now on, the upper indices $j$ 's of $\left\{\varphi^{j}, d \varphi^{j}, \cdots\right\}$ start from 1 to $l$ if we do not specify them.

\section{Moser-type Harnack Inequality}

In this section, we derive the Moser-type Harnack inequality ([M], [CC1]) for the total regularized energy density

$$
g:=f_{\delta}+\varepsilon e_{0}
$$

with

$$
f_{\delta}:=\left|\nabla_{b} \varphi\right|^{2}+\delta
$$

Let $\varphi:(M, J, \theta) \rightarrow\left(N, g_{i j}\right)$ be a map from $(M, J, \theta)$ to $\left(N, g_{i j}\right)$. We first derive the Euler-Lagrange equation associated to its $p$-energy $E_{p}(\varphi)$.

Lemma 3.1. Let $(M, J, \theta)$ be a closed pseudohermitian manifold and $\left(N^{m}, g\right)$ be a Riemannian manifold. $A C^{2} \operatorname{map} \varphi:(M, J, \theta) \rightarrow(N, g)$ is p-pseudoharmonic if and only if it satisfies the Euler-Lagrangian equations

$$
\triangle_{b, p} \varphi^{k}+2\left|\nabla_{b} \varphi\right|^{p-2} \widetilde{\Gamma}_{i j}^{k} \varphi_{\alpha}^{i} \varphi_{\bar{\alpha}}^{j}=0, \quad k=1, \ldots, m
$$

where $\triangle_{b, p} \varphi^{k}=\operatorname{div}_{b}\left(\left|\nabla_{b} \varphi\right|^{p-2} \nabla_{b} \varphi^{k}\right)$.

Proof. Let $\varphi_{t},-\varepsilon<t<\varepsilon$, be a smooth variation of $\varphi$ so that

$$
\varphi_{0}=\varphi \quad \text { and }\left.\quad \frac{d \varphi_{t}}{d t}\right|_{t=0}=V \in \Gamma\left(\varphi^{-1} T N\right) .
$$


$\varphi_{t}$ may be viewed as a map from $(-\varepsilon, \varepsilon) \times M$ into $N$. By direct computations, we have

$$
\begin{aligned}
& \frac{d E_{p}\left(\varphi_{t}\right)}{d t}=\frac{1}{p} \frac{d}{d t} \int_{M}\left|\nabla_{b} \varphi_{t}\right|^{p} d \mu \\
& =\frac{1}{2} \int_{M}\left|\nabla_{b} \varphi_{t}\right|^{p-2} \frac{d}{d t}\left|\nabla_{b} \varphi_{t}\right|^{2} d \mu=\int_{M}\left|\nabla_{b} \varphi_{t}\right|^{p-2} \frac{d}{d t}\left[g_{i j} \varphi_{t \alpha}^{i} \varphi_{t \bar{\alpha}}^{j}\right] d \mu \\
& =\int_{M}\left|\nabla_{b} \varphi_{t}\right|^{p-2}\left[g_{i j, k} \varphi_{t \alpha}^{i} \varphi_{t \bar{\alpha}}^{j} \frac{d \varphi_{t}^{k}}{d t}+g_{i j}\left(\frac{d \varphi_{t}^{i}}{d t}\right)_{t \alpha} \varphi_{t \bar{\alpha}}^{j}+g_{i j}\left(\frac{d \varphi_{t}^{j}}{d t}\right)_{\bar{\alpha}} \varphi_{\alpha}^{i}\right] d \mu \\
& =-\int_{M}\left[\left|\nabla_{b} \varphi_{t}\right|^{p-2} \triangle_{b} \varphi_{t}^{k}+\left\langle\nabla_{b}\left|\nabla_{b} \varphi_{t}\right|^{p-2}, \nabla_{b} \varphi_{t}^{k}\right\rangle+2\left|\nabla_{b} \varphi_{t}\right|^{p-2} \widetilde{\Gamma}_{i j}^{k} \varphi_{t \alpha}^{i} \varphi_{t \bar{\alpha}}^{j}\right] \frac{d \varphi_{t}^{k}}{d t} d \mu \\
& =-\int_{M}\left[\triangle_{b, p} \varphi_{t}^{k}+2\left|\nabla_{b} \varphi_{t}\right|^{p-2} \widetilde{\Gamma}_{i j}^{k} \varphi_{t \alpha}^{i} \varphi_{t \bar{\alpha}}^{j}\right] \frac{d \varphi_{t}^{k}}{d t} d \mu \\
& =-\int_{M}\left\langle\frac{d \varphi_{t}}{d t}, \tau_{p}\left(\varphi_{t}\right)\right\rangle d \mu .
\end{aligned}
$$

Thus, the first variational formula is given by

$$
\left.\frac{d}{d t} E_{p}\left(\varphi_{t}\right)\right|_{t=0}=-\int_{M}\left\langle V, \tau_{p}(\varphi)\right\rangle d \mu
$$

where $\tau_{p}(\varphi)$ is called the $p$-tension field of $\varphi$, which is defined by

$$
\tau_{p}(\varphi)=\sum_{k=1}^{m}\left[\triangle_{b, p} \varphi^{k}+2\left|\nabla_{b} \varphi\right|^{p-2} \widetilde{\Gamma}_{i j}^{k} \varphi_{\alpha}^{i} \varphi_{\bar{\alpha}}^{j}\right] \frac{\partial}{\partial y_{k}} .
$$

Therefore $\varphi \in C^{2}(M ; N)$ is a critical point of the $p$-energy functional $E_{p}(\varphi)$ if and only if its $p$-tension field $\tau_{p}(u)$ vanishes identically. That is, $\varphi$ is $p$-pseudoharmonic if and only if it satisfies the Euler-Lagrange equations (3.1).

Next we recall the $\mathrm{CR}$ version of Bochner formula for a real smooth function on a closed pseudohermitian manifold $\left(M^{2 n+1}, J, \theta\right)$.

Lemma 3.2. ([G]) Let $\left(M^{2 n+1}, J, \theta\right)$ be a closed pseudohermitian manifold. For a real smooth function $u$ on $(M, J, \theta)$,

$$
\begin{aligned}
\frac{1}{2} \Delta_{b}\left|\nabla_{b} u\right|^{2}= & \left|\left(\nabla^{H}\right)^{2} u\right|^{2}+\left\langle\nabla_{b} u, \nabla_{b} \Delta_{b} u\right\rangle_{L_{\theta}} \\
& +[2 \text { Ric }-(n-2) \text { Tor }]\left(\left(\nabla_{b} u\right)_{\mathbf{C}},\left(\nabla_{b} u\right)_{\mathbf{C}}\right)+2\left\langle J \nabla_{b} u, \nabla_{b} u_{0}\right\rangle_{L_{\theta}} .
\end{aligned}
$$


Here $\left(\nabla_{b} u\right)_{\mathbf{C}}=u_{\bar{\alpha}} Z_{\alpha}$ is the corresponding complex $(1,0)$-vector field of $\nabla_{b} u$ and $d_{b} u=$ $u_{\alpha} \theta^{\alpha}+u_{\bar{\alpha}} \theta^{\bar{\alpha}}$.

Since $f_{\delta}$ and $e_{0}(\varphi)$ are independent of the choice of local coordinates, for each point $(x, t)$ one may choose a normal coordinate chart $U$ at $(x, t)$ and a normal coordinate chart at $\varphi(x, t)$ such that $\varphi(U) \subset V$ and then fulfill the following computations at the point $(x, t)$.

Now we are ready to derive the Moser type Harnack inequality for the total regularized energy density.

Lemma 3.3. Let $\left(M^{2 n+1}, J, \theta\right)$ be a closed Sasakian manifold and $\left(N, g_{i j}\right)$ be a compact Riemannian manifold. The solution $\varphi$ of the regularized equation (1.5) satisfying the following inequalities:

(i) For $g=f_{\delta}+\varepsilon e_{0}=\left|\nabla_{b} \varphi\right|^{2}+\delta+\varepsilon e_{0}$,

$$
\begin{aligned}
& \frac{\partial g}{\partial t}-\operatorname{div}_{b}\left(f_{\delta}^{\frac{p-2}{2}} \nabla_{b} g\right)-(p-2) \operatorname{div}_{b}\left(f_{\delta}^{\frac{p-4}{2}}\left\langle\nabla_{b} f_{\delta}, \nabla_{b} \varphi^{k}\right\rangle \nabla_{b} \varphi^{k}\right) \\
& -2 \varepsilon \operatorname{div}_{b}\left(\left(f_{\delta}^{\frac{p-2}{2}}\right)_{0} \nabla_{b} \varphi^{k}\right) \varphi_{0}^{k}+\frac{p-2}{2} f_{\delta}^{\frac{p-4}{2}}\left|\nabla_{b} f_{\delta}\right|^{2}+2 f_{\delta}^{\frac{p-2}{2}}\left|\nabla_{b}^{2} \varphi^{k}\right|^{2}+2 \varepsilon f_{\delta}^{\frac{p-2}{2}}\left|\nabla_{b} \varphi_{0}^{k}\right|^{2} \\
& \leq C\left(f_{\delta}^{\frac{p}{2}}+f_{\delta}^{\frac{p+2}{2}}\right)+C \varepsilon f_{\delta}^{\frac{p}{2}} e_{0}-4 f_{\delta}^{\frac{p-2}{2}}\left\langle J \nabla_{b} \varphi^{k}, \nabla_{b} \varphi_{0}^{k}\right\rangle .
\end{aligned}
$$

(ii) If the sectional curvature of $\left(N, g_{i j}\right)$ is nonpositive

$$
K^{N} \leq 0
$$

then

$$
\begin{aligned}
& \frac{\partial g}{\partial t}-\operatorname{div}_{b}\left(f_{\delta}^{\frac{p-2}{2}} \nabla_{b} g\right)-(p-2) \operatorname{div}_{b}\left(f_{\delta}^{\frac{p-4}{2}}\left\langle\nabla_{b} f_{\delta}, \nabla_{b} \varphi^{k}\right\rangle \nabla_{b} \varphi^{k}\right) \\
& -2 \varepsilon \operatorname{div}_{b}\left(\left(f_{\delta}^{\frac{p-2}{2}}\right)_{0} \nabla_{b} \varphi^{k}\right) \varphi_{0}^{k}+\frac{p-2}{2} f_{\delta}^{\frac{p-4}{2}}\left|\nabla_{b} f_{\delta}\right|^{2}+2 f_{\delta}^{\frac{p-2}{2}}\left|\nabla_{b}^{2} \varphi^{k}\right|^{2}+2 \varepsilon f_{\delta}^{\frac{p-2}{2}}\left|\nabla_{b} \varphi_{0}^{k}\right|^{2} \\
& \leq C f_{\delta}^{\frac{p}{2}}-4 f_{\delta}^{\frac{p-2}{2}}\left\langle J \nabla_{b} \varphi^{k}, \nabla_{b} \varphi_{0}^{k}\right\rangle .
\end{aligned}
$$

By Young's inequality, the bad term $\left\langle J \nabla_{b} \varphi^{k}, \nabla_{b} \varphi_{0}^{k}\right\rangle$ in the RHS of (3.3) will be dominated by the good term $\left|\nabla_{b} \varphi_{0}\right|^{2}$ in LHS. As a consequence, we have 
Corollary 3.1. Let $\left(M^{2 n+1}, J, \theta\right)$ be a closed Sasakian manifold and $\left(N, g_{i j}\right)$ be a compact Riemannian manifold. The solution $\varphi$ of the regularized equation (1.5) satisfying the following inequalities:

(i) For $g=f_{\delta}+\varepsilon e_{0}=\left|\nabla_{b} \varphi\right|^{2}+\delta+\varepsilon e_{0}$,

$$
\begin{aligned}
& \frac{\partial g}{\partial t}-\operatorname{div}_{b}\left(f_{\delta}^{\frac{p-2}{2}} \nabla_{b} g\right)-(p-2) \operatorname{div}_{b}\left(f_{\delta}^{\frac{p-4}{2}}\left\langle\nabla_{b} f_{\delta}, \nabla_{b} \varphi^{k}\right\rangle \nabla_{b} \varphi^{k}\right. \\
& -2 \varepsilon \operatorname{div}_{b}\left(\left(f_{\delta}^{\frac{p-2}{2}}\right)_{0} \nabla_{b} \varphi^{k}\right) \varphi_{0}^{k}+\frac{p-2}{2} f_{\delta}^{\frac{p-4}{2}}\left|\nabla_{b} f_{\delta}\right|^{2}+2 f_{\delta}^{\frac{p-2}{2}}\left|\nabla_{b}^{2} \varphi^{k}\right|^{2}+\varepsilon f_{\delta}^{\frac{p-2}{2}}\left|\nabla_{b} \varphi_{0}^{k}\right|^{2} \\
& \leq C\left(f_{\delta}^{\frac{p}{2}}+f_{\delta}^{\frac{p+2}{2}}\right)+C \varepsilon f_{\delta}^{\frac{p}{2}} e_{0} .
\end{aligned}
$$

(ii) If the sectional curvature of $\left(N, g_{i j}\right)$ is nonpositive

$$
K^{N} \leq 0
$$

then

$$
\begin{aligned}
& \frac{\partial g}{\partial t}-\operatorname{div}_{b}\left(f_{\delta}^{\frac{p-2}{2}} \nabla_{b} g\right)-(p-2) \operatorname{div}_{b}\left(f_{\delta}^{\frac{p-4}{2}}\left\langle\nabla_{b} f_{\delta}, \nabla_{b} \varphi^{k}\right\rangle \nabla_{b} \varphi^{k}\right) \\
& -2 \varepsilon \operatorname{div}_{b}\left(\left(f_{\delta}^{\frac{p-2}{2}}\right)_{0} \nabla_{b} \varphi^{k}\right) \varphi_{0}^{k}+\frac{p-2}{2} f_{\delta}^{\frac{p-4}{2}}\left|\nabla_{b} f_{\delta}\right|^{2}+2 f_{\delta}^{\frac{p-2}{2}}\left|\nabla_{b}^{2} \varphi\right|^{2}+\varepsilon f_{\delta}^{\frac{p-2}{2}}\left|\nabla_{b} \varphi_{0}\right|^{2} \\
& \leq C f_{\delta}^{\frac{p}{2}}
\end{aligned}
$$

Proof. (i) We first compute $\frac{\partial}{\partial t} f_{\delta}-\operatorname{div}_{b}\left(f_{\delta}^{\frac{p-2}{2}} \nabla_{b} f_{\delta}\right)$ :

Note that $\left|\nabla_{b} \varphi\right|^{2}=2 g_{i j} \varphi_{\alpha}^{i} \varphi_{\bar{\alpha}}^{j}$. It is straightforward to compute as

$$
\begin{aligned}
\frac{\partial f_{\delta}}{\partial t}= & \frac{\partial}{\partial t}\left(2 g_{i j} \varphi_{\alpha}^{i} \varphi_{\bar{\alpha}}^{j}+\delta\right) \\
= & 2 g_{i j}\left(\frac{\partial \varphi^{i}}{\partial t}\right)_{\alpha} \varphi_{\bar{\alpha}}^{j}+2 g_{i j}\left(\frac{\partial \varphi^{j}}{\partial t}\right)_{\bar{\alpha}} \varphi_{\alpha}^{i} \\
= & 2 g_{k l}\left[\operatorname{div}\left(f_{\delta}^{\frac{p-2}{2}} \nabla_{b} \varphi^{k}\right)+2 f_{\delta}^{\frac{p-2}{2}} \tilde{\Gamma}_{i j}^{k} \varphi_{\alpha}^{i} \varphi_{\bar{\alpha}}^{j}\right]_{\beta} \varphi_{\bar{\beta}}^{l} \\
& +2 g_{k l}\left[\operatorname{div}\left(f_{\delta}^{\frac{p-2}{2}} \nabla_{b} \varphi^{k}\right)+2 f_{\delta}^{\frac{p-2}{2}} \tilde{\Gamma}_{i j}^{k} \varphi_{\alpha}^{i} \varphi_{\bar{\alpha}}^{j}\right]_{\bar{\beta}} \varphi_{\beta}^{l} \\
= & 2\left\langle\nabla_{b} \varphi^{k}, \nabla_{b} \operatorname{div}\left(f_{\delta}^{\frac{p-2}{2}} \nabla_{b} \varphi^{k}\right)\right\rangle \\
& +2 f_{\delta}^{\frac{p-2}{2}}\left[2 \widetilde{\Gamma}_{i j, l}^{k} \varphi_{\beta}^{l} \varphi_{\alpha}^{i} \varphi_{\bar{\alpha}}^{j} \varphi_{\bar{\beta}}^{k}+2 \widetilde{\Gamma}_{i j, l}^{k} \varphi_{\bar{\beta}}^{l} \varphi_{\alpha}^{i} \varphi_{\bar{\alpha}}^{j} \varphi_{\beta}^{k}\right]
\end{aligned}
$$


and from the CR Bochner formula (3.2)

$$
\begin{aligned}
\operatorname{div}_{b}\left(f_{\delta}^{\frac{p-2}{2}} \nabla_{b} f_{\delta}\right) \\
=\frac{p-2}{2} f_{\delta}^{\frac{p-4}{2}}\left|\nabla_{b} f_{\delta}\right|^{2}+f_{\delta}^{\frac{p-2}{2}} \triangle_{b} f_{\delta} \\
=\frac{p-2}{2} f_{\delta}^{\frac{p-4}{2}}\left|\nabla_{b} f_{\delta}\right|^{2}+2 f_{\delta}^{\frac{p-2}{2}} \triangle_{b}\left[g_{i j} \varphi_{\alpha}^{i} \varphi_{\bar{\alpha}}^{j}\right] \\
=\frac{p-2}{2} f_{\delta}^{\frac{p-4}{2}}\left|\nabla_{b} f_{\delta}\right|^{2}+2 f_{\delta}^{\frac{p-2}{2}}\left[\frac{1}{2} \triangle_{b}\left|\nabla_{b} \varphi^{k}\right|^{2}+\varphi_{\alpha}^{i} \varphi_{\bar{\alpha}} \triangle_{b} g_{i j}\right] \\
=\frac{p-2}{2} f_{\delta}^{\frac{p-4}{2}}\left|\nabla_{b} f_{\delta}\right|^{2}+2 f_{\delta}^{\frac{p-2}{2}}\left[\left|\nabla_{b}^{2} \varphi^{k}\right|^{2}+\left\langle\nabla_{b} \varphi^{k}, \nabla_{b} \triangle_{b} \varphi^{k}\right\rangle+\varphi_{\alpha}^{i} \varphi_{\bar{\alpha}} \triangle_{b} g_{i j}\right. \\
\left.\quad+(2 \operatorname{Ric}-(n-2) \operatorname{Tor})\left(\left(\nabla_{b} \varphi^{k}\right)_{C},\left(\nabla_{b} \varphi^{k}\right)_{C}\right)+2\left\langle J \nabla_{b} \varphi^{k}, \nabla_{b} \varphi_{0}^{k}\right\rangle\right]
\end{aligned}
$$

Thus, based on [CC1, (3.2) and (3.3)]

$$
\begin{aligned}
& \frac{\partial f_{\delta}}{\partial t}-\operatorname{div}_{b}\left(f_{\delta}^{\frac{p-2}{2}} \nabla_{b} f_{\delta}\right)-(p-2) \operatorname{div}_{b}\left(f_{\delta}^{\frac{p-4}{2}}\left\langle\nabla_{b} f_{\delta}, \nabla_{b} \varphi^{k}\right\rangle \nabla_{b} \varphi^{k}\right) \\
& +\frac{p-2}{2} f_{\delta}^{\frac{p-4}{2}}\left|\nabla_{b} f_{\delta}\right|^{2}+2 f_{\delta}^{\frac{p-2}{2}}\left|\nabla_{b}^{2} \varphi\right|^{2} \\
& =2 f_{\delta}^{\frac{p-2}{2}}\left[2 \widetilde{\Gamma}_{i j, l}^{k} \varphi_{\beta}^{l} \varphi_{\alpha}^{i} \varphi_{\bar{\alpha}}^{j} \varphi_{\bar{\beta}}^{k}+2 \widetilde{\Gamma}_{i j, l}^{k} \varphi_{\bar{\beta}}^{l} \varphi_{\alpha}^{i} \varphi_{\bar{\alpha}}^{j} \varphi_{\beta}^{k}-\varphi_{\alpha}^{i} \varphi_{\bar{\alpha}} \triangle_{b} g_{i j}\right. \\
& \left.-(2 \operatorname{Ric}-(n-2) \operatorname{Tor})\left(\left(\nabla_{b} \varphi^{k}\right)_{C},\left(\nabla_{b} \varphi^{k}\right)_{C}\right)\right]-4 f_{\delta}^{\frac{p-2}{2}}\left\langle J \nabla_{b} \varphi^{k}, \nabla_{b} \varphi_{0}^{k}\right\rangle \\
& =2 f_{\delta}^{\frac{p-2}{2}}\left[2 \tilde{R}_{i j k l} \varphi_{\alpha}^{i} \varphi_{\beta}^{j} \varphi_{\bar{\alpha}}^{k} \varphi_{\bar{\beta}}^{l}+2 \tilde{R}_{i j k l} \varphi_{\alpha}^{i} \varphi_{\bar{\beta}}^{j} \varphi_{\bar{\alpha}}^{k} \varphi_{\beta}^{l}\right. \\
& \left.\left.-(2 R i c-(n-2) T o r)\left(\left(\nabla_{b} \varphi^{k}\right)_{C},\left(\nabla_{b} \varphi^{k}\right)_{C}\right)\right]\right]-4 f_{\delta}^{\frac{p-2}{2}}\left\langle J \nabla_{b} \varphi^{k}, \nabla_{b} \varphi_{0}^{k}\right\rangle \\
& \leq C f_{\delta}^{\frac{p}{2}}+C f_{\delta}^{\frac{p+2}{2}}-4 f_{\delta}^{\frac{p-2}{2}}\left\langle J \nabla_{b} \varphi^{k}, \nabla_{b} \varphi_{0}^{k}\right\rangle .
\end{aligned}
$$

This implies

$$
\begin{aligned}
& \frac{\partial f_{\delta}}{\partial t}-\operatorname{div}_{b}\left(f_{\delta}^{\frac{p-2}{2}} \nabla_{b} f_{\delta}\right)-(p-2) \operatorname{div}_{b}\left(f_{\delta}^{\frac{p-4}{2}}\left\langle\nabla_{b} f_{\delta}, \nabla_{b} \varphi^{k}\right\rangle \nabla_{b} \varphi^{k}\right) \\
& +\frac{p-2}{2} f_{\delta}^{\frac{p-4}{2}}\left|\nabla_{b} f_{\delta}\right|^{2}+2 f_{\delta}^{\frac{p-2}{2}}\left|\nabla_{b}^{2} \varphi^{k}\right|^{2} \\
& \leq C f_{\delta}^{\frac{p}{2}}+C f_{\delta}^{\frac{p+2}{2}}-4 f_{\delta}^{\frac{p-2}{2}}\left\langle J \nabla_{b} \varphi^{k}, \nabla_{b} \varphi_{0}^{k}\right\rangle .
\end{aligned}
$$

Next we compute $\frac{\partial}{\partial t} e_{0}(\varphi)-\operatorname{div}_{b}\left(f_{\delta}^{\frac{p-2}{2}} \nabla_{b} e_{0}(\varphi)\right)$ : 
We observe from ([CC2]) that for any smooth function $u$,

$$
\left[\Delta_{b}, T\right] u=4\left[i \sum_{\alpha, \beta=1}^{n}\left(A_{\bar{\alpha} \bar{\beta}} u_{\beta}\right)_{\alpha}\right]
$$

Thus for any Sasakian manifold $\left(M^{2 n+1}, J, \theta\right)$ (i.e. vanishing pseudohermitian torsion),

$$
\left[\Delta_{b}, T\right] u=0
$$

We first compute

$$
\frac{\partial e_{0}(\varphi)}{\partial t}=2 g_{i j}\left(\frac{\partial \varphi^{i}}{\partial t}\right)_{0} \varphi_{0}^{j}=2 g_{k l}\left[\operatorname{div}_{b}\left(f_{\delta}^{\frac{p-2}{2}} \nabla_{b} \varphi^{k}\right)+2 f_{\delta}^{\frac{p-2}{2}} \tilde{\Gamma}_{i j}^{k} \varphi_{\alpha}^{i} \varphi_{\bar{\alpha}}^{j}\right]_{0} \varphi_{0}^{l}
$$

and

$$
\operatorname{div}_{b}\left(f_{\delta}^{\frac{p-2}{2}} \nabla_{b} e_{0}(\varphi)\right)=\left\langle\nabla_{b} f_{\delta}^{\frac{p-2}{2}}, \nabla_{b} e_{0}\right\rangle+f_{\delta}^{\frac{p-2}{2}}\left[2 \varphi_{0}^{i} \triangle_{b} \varphi_{0}^{i}+2\left|\nabla_{b} \varphi_{0}^{i}\right|^{2}+\varphi_{0}^{i} \varphi_{0}^{j} \triangle_{b} g_{i j}\right]
$$

Based on [CC1, (3.4)] and (3.7), one can derive

$$
\begin{aligned}
& \frac{\partial}{\partial t} e_{0}(\varphi)-\operatorname{div}_{b}\left(f_{\delta}^{\frac{p-2}{2}} \nabla_{b} e_{0}(\varphi)\right)-2 \operatorname{div}_{b}\left(\left(f_{\delta}^{\frac{p-2}{2}}\right)_{0} \nabla_{b} \varphi^{k}\right) \varphi_{0}^{k} \\
& =f_{\delta}^{\frac{p-2}{2}}\left[4 \widetilde{\Gamma}_{i j, l}^{k} \varphi_{\alpha}^{i} \varphi_{\bar{\alpha}}^{j} \varphi_{0}^{k} \varphi_{0}^{l}-\varphi_{0}^{i} \varphi_{0}^{j} \triangle_{b} g_{i j}\right]-2 f_{\delta}^{\frac{p-2}{2}}\left|\nabla_{b} \varphi_{0}\right|^{2}
\end{aligned}
$$

On the other hand ( [CC1, (3.5)]),

$$
4 \sum_{i, j, k, \ell=1}^{m} \sum_{\alpha=1}^{n} \widetilde{\Gamma}_{i j, l}^{k} \varphi_{\alpha}^{i} \varphi_{\bar{\alpha}}^{j} \varphi_{0}^{k} \varphi_{0}^{\ell}-\sum_{i, j=1}^{m} \varphi_{0}^{i} \varphi_{0}^{j} \Delta_{b}\left(g_{i j}\right)=4 \sum_{i, j, k, \ell=1}^{m} \sum_{\alpha=1}^{n} \widetilde{R}_{i j k \ell} \varphi_{\alpha}^{i} \varphi_{0}^{j} \varphi_{\alpha}^{k} \varphi_{0}^{\ell} .
$$

Hence

$$
\begin{aligned}
& \frac{\partial}{\partial t} e_{0}(\varphi)-\operatorname{div}_{b}\left(f_{\delta}^{\frac{p-2}{2}} \nabla_{b} e_{0}(\varphi)\right)-2 \operatorname{div}_{b}\left(\left(f_{\delta}^{\frac{p-2}{2}}\right)_{0} \nabla_{b} \varphi^{k}\right) \varphi_{0}^{k} \\
& =f_{\delta}^{\frac{p-2}{2}}\left[4 \widetilde{R}_{i j k \ell} \varphi_{\alpha}^{i} \varphi_{0}^{j} \varphi_{\frac{k}{\alpha}} \varphi_{0}^{\ell}\right]-2 f_{\delta}^{\frac{p-2}{2}}\left|\nabla_{b} \varphi_{0}\right|^{2} \\
& \leq C f_{\delta}^{\frac{p}{2}} e_{0}-2 f_{\delta}^{\frac{p-2}{2}}\left|\nabla_{b} \varphi_{0}\right|^{2} .
\end{aligned}
$$


From (3.6) and (3.8), we have

$$
\begin{aligned}
& \frac{\partial g}{\partial t}-\operatorname{div}_{b}\left(f_{\delta}^{\frac{p-2}{2}} \nabla_{b} g\right)-(p-2) \operatorname{div}_{b}\left(f_{\delta}^{\frac{p-4}{2}}\left\langle\nabla_{b} f_{\delta}, \nabla_{b} \varphi^{k}\right\rangle \nabla_{b} \varphi^{k}\right) \\
& -2 \varepsilon \operatorname{div}_{b}\left(\left(f_{\delta}^{\frac{p-2}{2}}\right)_{0} \nabla_{b} \varphi^{k}\right) \varphi_{0}^{k}+\frac{p-2}{2} f_{\delta}^{\frac{p-4}{2}}\left|\nabla_{b} f_{\delta}\right|^{2}+2 f_{\delta}^{\frac{p-2}{2}}\left|\nabla_{b}^{2} \varphi\right|^{2}+2 \varepsilon f_{\delta}^{\frac{p-2}{2}}\left|\nabla_{b} \varphi_{0}\right|^{2} \\
& \leq C\left(f_{\delta}^{\frac{p}{2}}+f_{\delta}^{\frac{p+2}{2}}\right)+C \varepsilon f_{\delta}^{\frac{p}{2}} e_{0}-4 f_{\delta}^{\frac{p-2}{2}}\left\langle J \nabla_{b} \varphi^{k}, \nabla_{b} \varphi_{0}^{k}\right\rangle .
\end{aligned}
$$

(ii) However, if the sectional curvature of $\left(N, g_{i j}\right)$ is nonpositive

$$
K^{N} \leq 0
$$

it follows from (3.5) and (3.8) that

$$
\begin{aligned}
& \frac{\partial f_{\delta}}{\partial t}-\operatorname{div}_{b}\left(f_{\delta}^{\frac{p-2}{2}} \nabla_{b} f_{\delta}\right)-(p-2) \operatorname{div}_{b}\left(f_{\delta}^{\frac{p-4}{2}}\left\langle\nabla_{b} f_{\delta}, \nabla_{b} \varphi^{k}\right\rangle \nabla_{b} \varphi^{k}\right) \\
& +\frac{p-2}{2} f_{\delta}^{\frac{p-4}{2}}\left|\nabla_{b} f_{\delta}\right|^{2}+2 f_{\delta}^{\frac{p-2}{2}}\left|\nabla_{b}^{2} \varphi^{k}\right|^{2} \\
& \leq C f_{\delta}^{\frac{p}{2}}-4 f_{\delta}^{\frac{p-2}{2}}\left\langle J \nabla_{b} \varphi^{k}, \nabla_{b} \varphi_{0}^{k}\right\rangle .
\end{aligned}
$$

and

$$
\begin{aligned}
& \frac{\partial}{\partial t} e_{0}(\varphi)-\operatorname{div}_{b}\left(f_{\delta}^{\frac{p-2}{2}} \nabla_{b} e_{0}(\varphi)\right)-2 \operatorname{div}_{b}\left(\left(f_{\delta}^{\frac{p-2}{2}}\right)_{0} \nabla_{b} \varphi^{k}\right) \varphi_{0}^{k} \\
& \leq-2 f_{\delta}^{\frac{p-2}{2}}\left|\nabla_{b} \varphi_{0}\right|^{2} .
\end{aligned}
$$

All these imply

$$
\begin{aligned}
& \frac{\partial g}{\partial t}-\operatorname{div}_{b}\left(f_{\delta}^{\frac{p-2}{2}} \nabla_{b} g\right)-(p-2) \operatorname{div}_{b}\left(f_{\delta}^{\frac{p-4}{2}}\left\langle\nabla_{b} f_{\delta}, \nabla_{b} \varphi^{k}\right\rangle \nabla_{b} \varphi^{k}\right) \\
& -2 \varepsilon \operatorname{div}_{b}\left(\left(f_{\delta}^{\frac{p-2}{2}}\right)_{0} \nabla_{b} \varphi^{k}\right) \varphi_{0}^{k}+\frac{p-2}{2} f_{\delta}^{\frac{p-4}{2}}\left|\nabla_{b} f_{\delta}\right|^{2}+2 f_{\delta}^{\frac{p-2}{2}}\left|\nabla_{b}^{2} \varphi\right|^{2}+2 \varepsilon f_{\delta}^{\frac{p-2}{2}}\left|\nabla_{b} \varphi_{0}\right|^{2} \\
& \leq C f_{\delta}^{\frac{p}{2}}-4 f_{\delta}^{\frac{p-2}{2}}\left\langle J \nabla_{b} \varphi^{k}, \nabla_{b} \varphi_{0}^{k}\right\rangle .
\end{aligned}
$$

This completes the proof of this lemma. 


\section{Proof of Main Results}

In this section, we will prove a uniform estimate for the $p$-energy density and then the global existence and asymptotic convergence of the $p$-pseudoharmonic map heat flow.

Lemma 4.1. Let $\left(M^{2 n+1}, J, \theta\right)$ be a closed Sasakian manifold and $\left(N, g_{i j}\right)$ be a compact Riemannian manifold. Let $u_{0} \in C^{2, \alpha}(M, N), 0<\alpha<1,\left\|\nabla u_{0}\right\|_{L^{\infty}(M)} \leq K$ and $\varphi_{\delta}$ is the solution of the regularized equation (1.5).

(i) For $g=\left|\nabla_{b} \varphi_{\delta}\right|^{2}+\delta+\varepsilon e_{0}$ and all $q \geq \frac{p}{2}$, there exists $\varepsilon_{1}>0$ depending on $M, N$ and $q$ such that if

$$
\sup _{0 \leq t<T^{\prime}}\|g(t, \cdot)\|_{L^{n+1}(M)} \leq \varepsilon_{1}
$$

then

$$
\sup _{1 \leq t_{1} \leq T^{\prime}}\|g\|_{L^{q}\left(\left[t_{1}-1, t_{1}\right] \times M\right)} \leq C, \quad \text { when } \quad T^{\prime}>1
$$

and

$$
\|g\|_{L^{q}\left(\left[0, T^{\prime}\right) \times M\right)} \leq C, \text { when } \quad T^{\prime} \leq 1,
$$

where $C$ is a constant depending on $K, M, N$ and $q$.

(ii) In addition, if the sectional curvature of $\left(N, g_{i j}\right)$ is nonpositive

$$
K^{N} \leq 0
$$

then (4.2) and (4.3) hold without assumption (4.1).

Proof. (i) Let us first prove (4.2). Fix $t_{0} \geq 1$ and $x_{0} \in M$. Let $\rho<R \leq R_{0}=\inf \left(R_{M}, 1\right)$ where $R_{M}$ denotes the radius of ball on which CR Sobolev inequality (4.22) holds and set $Q_{R}=\left(t_{0}-R, t_{0}\right) \times B\left(x_{0}, R\right)$. We choose $\psi \in C_{0}\left(B\left(x_{0}, R\right)\right)$ such that $\psi=1, B\left(x_{0}, \rho\right) ; 0 \leq$ $\psi \leq 1 ;\left|\nabla_{b} \psi\right| \leq c(R-\rho)^{-1}$, and let $\eta \in C^{\infty}(R)$ with $\eta(t)=0, \quad t \leq t_{0}-R ; \eta(t)=1, t \geq$ 
$t_{0}-\rho ; 0 \leq \eta(t) \leq 1 ; \quad\left|\eta^{\prime}\right| \leq c(R-\rho)^{-1}$. We set $\phi(x, t)=\psi(x) \eta(t)$. Multiply inequality (3.3) by $g^{r} \phi^{k}, r \geq 0, k \in N$, and integrate on $\left[t_{0}-R, t\right] \times B\left(x_{0}, R\right), t_{0}-R<t \leq t_{0}$, we have

$$
\begin{aligned}
& \frac{1}{r+1} \sup _{t \leq t_{0}} \int_{B_{x_{0}}(R)} g^{r+1} \phi^{k} d \mu+r \int_{Q_{R}} f_{\delta}^{\frac{p-2}{2}} g^{r-1} \phi^{k}\left|\nabla_{b} g\right|^{2} d \mu d t \\
& +(p-2) r \int_{Q_{R}} f_{\delta}^{\frac{p-4}{2}}\left\langle\nabla_{b} f_{\delta}, \nabla_{b} \varphi^{l}\right\rangle^{2} g^{r-1} \phi^{k} d \mu d t+(p-2) \varepsilon \int_{Q_{R}} f_{\delta}^{\frac{p-4}{2}}\left(f_{\delta}\right)_{0}^{2} g^{r} \phi^{k} d \mu d t \\
& +\frac{p-2}{2} \int_{Q_{R}} f_{\delta}^{\frac{p-4}{2}} g^{r} \phi^{k}\left|\nabla_{b} f_{\delta}\right|^{2} d \mu d t+2 \int_{Q_{R}} f_{\delta}^{\frac{p-2}{2}}\left|\nabla_{b}^{2} \varphi^{l}\right|^{2} g^{r} \phi^{k} d \mu d t \\
& +2 \varepsilon \int_{Q_{R}} f_{\delta}^{\frac{p-2}{2}} g^{r} \phi^{k}\left|\nabla_{b} \varphi_{0}^{l}\right|^{2} d \mu d t \\
& \leq \frac{k}{1+r} \int_{Q_{R}} g^{r+1} \phi^{k-1}\left|\frac{\partial \phi}{\partial t}\right| d \mu d t-k \int_{Q_{R}} f_{\delta}^{\frac{p-2}{2}} g^{r} \phi^{k-1}\left\langle\nabla_{b} g, \nabla_{b} \phi\right\rangle d \mu d t \\
& -(p-2) k \int_{Q_{R}} f_{\delta}^{\frac{p-4}{2}}\left\langle\nabla_{b} f_{\delta}, \nabla_{b} \varphi^{l}\right\rangle\left\langle\nabla_{b} \varphi^{l}, \nabla_{b} \phi\right\rangle \phi^{k-1} g^{r} d \mu d t \\
& -(p-2) k \varepsilon \int_{Q_{R}} f_{\delta}^{\frac{p-4}{2}}\left(f_{\delta}\right)_{0} \varphi_{0}^{l} g^{r} \phi^{k-1}\left\langle\nabla_{b} \varphi^{l}, \nabla_{b} \phi\right\rangle d \mu d t \\
& -(p-2) r \varepsilon \int_{Q_{R}} f_{\delta}^{\frac{p-4}{2}}\left(f_{\delta}\right)_{0} g^{r-1} \varphi_{0}^{l} \phi^{k}\left\langle\nabla_{b} \varphi^{l}, \nabla_{b} g\right\rangle d \mu d t \\
& -(p-2) r \varepsilon \int_{Q_{R}} f_{\delta}^{\frac{p-4}{2}}\left\langle\nabla_{b} f_{\delta}, \nabla_{b} \varphi^{l}\right\rangle\left\langle\nabla_{b} \varphi^{l}, \nabla_{b} e_{0}\right\rangle g^{r-1} \phi^{k} d \mu d t \\
& +C \int_{Q_{R}}\left(f_{\delta}^{\frac{p}{2}}+f_{\delta}^{\frac{p+2}{2}}+\varepsilon f_{\delta}^{\frac{p}{2}} e_{0}\right) g^{r} \phi^{k} d \mu d t-4 \int_{Q_{R}} f_{\delta}^{\frac{p-2}{2}} g^{r} \phi^{k}\left\langle J \nabla_{b} \varphi^{l}, \nabla_{b} \varphi_{0}^{l}\right\rangle d \mu d t
\end{aligned}
$$

By using Young's inequalities, we have

$$
\begin{aligned}
& -(p-2) r \varepsilon \int_{Q_{R}} f_{\delta}^{\frac{p-4}{2}}\left\langle\nabla_{b} f_{\delta}, \nabla_{b} \varphi^{l}\right\rangle\left\langle\nabla_{b} \varphi^{l}, \nabla_{b} e_{0}\right\rangle g^{r-1} \phi^{k} d \mu d t \\
& \leq \frac{(p-2) r}{4} \int_{Q_{R}} f_{\delta}^{\frac{p-4}{2}}\left\langle\nabla_{b} f_{\delta}, \nabla_{b} \varphi^{l}\right\rangle^{2} g^{r-1} \phi^{k} d \mu \\
& +(p-2) r \varepsilon^{2} \int_{Q_{R}} f_{\delta}^{\frac{p-4}{2}}\left\langle\nabla_{b} \varphi^{l}, \nabla_{b} e_{0}\right\rangle^{2} g^{r-1} \phi^{k} d \mu d t \\
& \leq \frac{(p-2) r}{4} \int_{Q_{R}} f_{\delta}^{\frac{p-4}{2}}\left\langle\nabla_{b} f_{\delta}, \nabla_{b} \varphi^{l}\right\rangle^{2} g^{r-1} \phi^{k} d \mu \\
& +4(p-2) r \varepsilon^{2} \int_{Q_{R}} f_{\delta}^{\frac{p-2}{2}}\left|\nabla_{b} \varphi_{0}^{l}\right|^{2} e_{0} g^{r-1} \phi^{k} d \mu d t
\end{aligned}
$$


and

$$
\begin{aligned}
& -(p-2) r \varepsilon \int_{Q_{R}} f_{\delta}^{\frac{p-4}{2}}\left(f_{\delta}\right)_{0} g^{r-1} \varphi_{0}^{l} \phi^{k}\left\langle\nabla_{b} \varphi^{l}, \nabla_{b} g\right\rangle d \mu d t \\
& =-(p-2) r \varepsilon \int_{Q_{R}} f_{\delta}^{\frac{p-4}{2}}\left(f_{\delta}\right)_{0} g^{r-1} \varphi_{0}^{l}\left\langle\nabla_{b} \varphi^{l}, \nabla_{b} f_{\delta}\right\rangle \phi^{k} d \mu d t \\
& -(p-2) r \varepsilon \int_{Q_{R}} f_{\delta}^{\frac{p-4}{2}}\left(f_{\delta}\right)_{0} g^{r-1} \varphi_{0}^{l}\left\langle\nabla_{b} \varphi^{l}, \varepsilon \nabla_{b} e_{0}\right\rangle \phi^{k} d \mu d t \\
& \leq \frac{(p-2) \varepsilon}{4} \int_{Q_{R}} f_{\delta}^{\frac{p-4}{2}}\left(f_{\delta}\right)_{0}^{2} g^{r} \phi^{k} d \mu+(p-2) r^{2} \varepsilon \int_{Q_{R}} f_{\delta}^{\frac{p-4}{2}} g^{r-2} e_{0}\left\langle\nabla_{b} \varphi^{l}, \nabla_{b} f_{\delta}\right\rangle^{2} \phi^{k} d \mu d t \\
& +\frac{(p-2) \varepsilon}{4} \int_{M} f_{\delta}^{\frac{p-4}{2}}\left(f_{\delta}\right)_{0}^{2} g^{r} \phi^{k} d \mu+(p-2) r^{2} \varepsilon^{3} \int_{M} f_{\delta}^{\frac{p-4}{2}} g^{r-2} e_{0} f_{\delta}\left|\nabla_{b} \varphi_{0}^{l}\right|^{2} e_{0}^{2} \phi^{k} d \mu d t \\
& =\frac{(p-2) \varepsilon}{2} \int_{M} f_{\delta}^{\frac{p-4}{2}}\left(f_{\delta}\right)_{0}^{2} g^{r} \phi^{k} d \mu+(p-2) r^{2} \varepsilon \int_{Q_{R}} f_{\delta}^{\frac{p-4}{2}} g^{r-2} e_{0}\left\langle\nabla_{b} \varphi^{l}, \nabla_{b} f_{\delta}\right\rangle^{2} \phi^{k} d \mu d t \\
& +(p-2) r^{2} \varepsilon^{3} \int_{M} f_{\delta}^{\frac{p-4}{2}} g^{r-2} e_{0} f_{\delta}\left|\nabla_{b} \varphi_{0}^{l}\right|^{2} e_{0}^{2} \phi^{k} d \mu d t
\end{aligned}
$$

and

$$
\begin{aligned}
& -(p-2) k \varepsilon \int_{Q_{R}} f_{\delta}^{\frac{p-4}{2}}\left(f_{\delta}\right)_{0} \varphi_{0}^{l} g^{r} \phi^{k-1}\left\langle\nabla_{b} \varphi^{l}, \nabla_{b} \phi\right\rangle d \mu d t \\
& \leq \frac{(p-2) \varepsilon}{4} \int_{Q_{R}} f_{\delta}^{\frac{p-4}{2}}\left(f_{\delta}\right)_{0}^{2} g^{r} \phi^{k} d \mu+(p-2) k^{2} \varepsilon \int_{Q_{R}} f_{\delta}^{\frac{p-4}{2}} g^{r} e_{0} f_{\delta}\left|\nabla_{b} \phi\right|^{2} \phi^{k-2} d \mu d t
\end{aligned}
$$

and

$$
\begin{aligned}
& -(p-2) k \int_{Q_{R}} f_{\delta}^{\frac{p-4}{2}}\left\langle\nabla_{b} f_{\delta}, \nabla_{b} \varphi^{l}\right\rangle\left\langle\nabla_{b} \varphi^{l}, \nabla_{b} \phi\right\rangle \phi^{k-1} g^{r} d \mu d t \\
& \leq(p-2) \int_{Q_{R}} f_{\delta}^{\frac{p-4}{2}} g^{r-1}\left\langle\nabla_{b} f_{\delta}, \nabla_{b} \varphi^{l}\right\rangle^{2} \phi^{k} d \mu d t \\
& +(p-2) \frac{k^{2}}{4} \int_{Q_{R}} f_{\delta}^{\frac{p-4}{2}} g^{r+1} f_{\delta}\left|\nabla_{b} \phi\right|^{2} \phi^{k-2} d \mu d t
\end{aligned}
$$

and

$$
\begin{aligned}
& -k \int_{Q_{R}} f_{\delta}^{\frac{p-2}{2}} g^{r} \phi^{k-1}\left\langle\nabla_{b} g, \nabla_{b} \phi\right\rangle d \mu d t \\
& \leq \frac{r}{2} \int_{Q_{R}} f_{\delta}^{\frac{p-2}{2}} g^{r-1}\left|\nabla_{b} g\right|^{2} \phi^{k} d \mu d t+\frac{2 k^{2}}{r} \int_{Q_{R}} \int_{Q_{R}} f_{\delta}^{\frac{p-2}{2}} g^{r+1}\left|\nabla_{b} \phi\right|^{2} \phi^{k-2} d \mu d t
\end{aligned}
$$


and

$$
\begin{aligned}
& -4 \int_{Q_{R}} f_{\delta}^{\frac{p-2}{2}} g^{r} \phi^{k}\left\langle J \nabla_{b} \varphi^{l}, \nabla_{b} \varphi_{0}^{l}\right\rangle d \mu d t \\
& \leq \frac{\varepsilon}{4} \int_{Q_{R}} f_{\delta}^{\frac{p-2}{2}} g^{r} \phi^{k}\left|\nabla_{b} \varphi_{0}^{l}\right|^{2} d \mu d t+\frac{16}{\varepsilon} \int_{Q_{R}} f_{\delta}^{\frac{p-2}{2}} g^{r} \phi^{k} f_{\delta} d \mu d t .
\end{aligned}
$$

From (4.4)-(4.10), we have

$$
\begin{aligned}
& \frac{1}{1+r} \sup _{t \leq t_{0}} \int_{B\left(x_{0}, R\right)} g^{1+r} \phi^{k} d \mu+\frac{r}{2} \int_{Q_{R}} f_{\delta}^{\frac{p-2}{2}} g^{r-1}\left|\nabla_{b} g\right|^{2} \phi^{k} d \mu d t \\
& +(p-2)\left(\frac{3 r}{4}-1\right) \int_{Q_{R}} f_{\delta}^{\frac{p-4}{2}} g^{r-1}\left\langle\nabla_{b} f_{\delta}, \nabla_{b} \varphi^{l}\right\rangle^{2} \phi^{k} d \mu d t \\
& -(p-2) r^{2} \varepsilon \int_{Q_{R}} f_{\delta}^{\frac{p-4}{2}} g^{r-2} e_{0}\left\langle\nabla_{b} \varphi^{l}, \nabla_{b} f_{\delta}\right\rangle^{2} \phi^{k} d \mu d t \\
& +\frac{p-2}{2} \int_{Q_{R}} f_{\delta}^{\frac{p-4}{2}} g^{r} \phi^{k}\left|\nabla_{b} f_{\delta}\right|^{2} d \mu d t+2 \int_{Q_{R}} f_{\delta}^{\frac{p-2}{2}}\left|\nabla_{b}^{2} \varphi^{l}\right|^{2} g^{r} \phi^{k} d \mu d t \\
& +\frac{7 \varepsilon}{4} \int_{Q_{R}} f_{\delta}^{\frac{p-2}{2}}\left|\nabla_{b} \varphi_{0}^{l}\right|^{2} g^{r} \phi^{k} d \mu d t-(p-2) r^{2} \varepsilon^{3} \int_{Q_{R}} f_{\delta}^{\frac{p-2}{2}} g^{r-2} e_{0}^{2}\left|\nabla_{b} \varphi_{0}^{l}\right|^{2} \phi^{k} d \mu d t \\
& -4(p-2) r \varepsilon^{2} \int_{Q_{R}} f_{\delta}^{\frac{p-2}{2}}\left|\nabla_{b} \varphi_{0}^{l}\right|^{2} e_{0} g^{r-1} \phi^{k} d \mu d t \\
& \leq \frac{k}{1+r} \int_{Q_{R}} g^{1+r} \phi^{k-1}\left|\frac{\partial \phi}{\partial t}\right| d \mu d t+(p-2) k^{2} \varepsilon \int_{Q_{R}} f_{\delta}^{\frac{p-2}{2}} g^{r} e_{0}\left|\nabla_{b} \phi\right|^{2} \phi^{k-2} d \mu d t \\
& +\frac{2 k^{2}}{r} \int_{Q_{R}} f_{\delta}^{\frac{p-2}{2}} g^{1+r}\left|\nabla_{b} \phi\right|^{2} \phi^{k-2} d \mu d t+(p-2) \frac{k^{2}}{4} \int_{Q_{R}}^{\frac{p-2}{2}} g^{1+r}\left|\nabla_{b} \phi\right|^{2} \phi^{k-2} d \mu d t \\
& +\frac{16}{\varepsilon} \int_{Q_{R}} f_{\delta}^{\frac{p}{2}} g^{r} \phi^{k} d \mu d t+C \int_{Q_{R}}^{\frac{p}{2}}\left[f_{\delta}^{\frac{p}{2}}+f_{\delta}^{\frac{p+2}{2}}+\varepsilon f_{\delta}^{r} e_{0}\right] g^{k} d \mu d t .
\end{aligned}
$$

For any fixed $t$, then we choose $\frac{1}{r^{4}}<\varepsilon<\frac{1}{r^{3}}$ such that

$$
\left(r^{2}-c\right) \varepsilon e_{0} \leq c f_{\delta}
$$

for some positive constant $c$ and

$$
\varepsilon r^{2} e_{0} \leq c f_{\delta}+c \varepsilon e_{0}
$$

That is

$$
\frac{\varepsilon e_{0}}{f_{\delta}+\varepsilon e_{0}} \leq \frac{c}{r^{2}} \leq \frac{\frac{3 r}{4}-1}{r^{2}}
$$


for some $r$ is large enough and then

$$
\left(\frac{3 r}{4}-1\right) g-r^{2} \varepsilon e_{0} \geq 0
$$

From (4.12), we have

$$
f_{\delta} \geq \frac{e_{0}}{r^{2}} \geq \varepsilon e_{0}
$$

so we have

$$
f_{\delta}=\frac{1}{2} f_{\delta}+\frac{1}{2} f_{\delta} \geq \frac{1}{2} f_{\delta}+\frac{1}{2} \varepsilon e_{0}=\frac{1}{2} g
$$

and

$$
\frac{7}{4}\left(f_{\delta}+\varepsilon e_{0}\right)^{2}-(p-2) r^{2} \varepsilon^{2} e_{0}^{2}-4(p-2) \varepsilon e_{0}\left(f_{\delta}+\varepsilon e_{0}\right) \geq 0 .
$$

From (4.11)-(4.15),

$$
\begin{aligned}
& \sup _{t \leq t_{0}} \int_{B\left(x_{0}, R\right)} g^{1+r} \phi^{k} d \mu+\int_{Q_{R}} g^{r+\frac{p}{2}-2}\left|\nabla_{b} g\right|^{2} \phi^{k} d \mu d t \\
& \leq C \int_{Q_{R}} g^{1+r} \phi^{k-1}\left|\frac{\partial \phi}{\partial t}\right| d \mu d t+C \int_{Q_{R}} g^{r+\frac{p}{2}} \phi^{k-2}\left|\nabla_{b} \phi\right|^{2} d \mu d t \\
& +C \int_{Q_{R}}\left(g^{r+\frac{p}{2}}+g^{r+\frac{p}{2}+1}\right) \phi^{k} d \mu d t,
\end{aligned}
$$

where $C$ is a positive constant depending on $k, M, N, r, \varepsilon, p$. Thus

$$
\begin{aligned}
& \sup _{t \leq t_{0}} \int_{B\left(x_{0}, R\right)} g^{1+r} \phi^{k} d \mu+\int_{Q_{R}} g^{r+\frac{p}{2}-2}\left|\nabla_{b} g\right|^{2} \phi^{k} d \mu d t \\
& \leq C \int_{Q_{R}} g^{1+r} \phi^{k-1}\left|\frac{\partial \phi}{\partial t}\right| d \mu d t+C \int_{Q_{R}} g^{r+\frac{p}{2}} \phi^{k-2}\left(\left|\nabla_{b} \phi\right|^{2}+\phi^{2}\right) d \mu d t \\
& +C \int_{Q_{R}} g^{r+\frac{p}{2}+1} \phi^{k} d \mu d t .
\end{aligned}
$$


Let $h=\frac{r+\frac{p}{2}+1}{r+1}$ and $l=\frac{r+\frac{p}{2}+1}{\frac{p}{2}}$ and fix $k \geq l$ large enough. By Hölder's inequality and Young's inequality, we have

$$
\begin{aligned}
& \int_{Q_{R}} g^{1+r} \phi^{k-1}\left|\frac{\partial \phi}{\partial t}\right| d \mu d t \leq\left(\int_{Q_{R}} g^{r+\frac{p}{2}+1} \phi^{k} d \mu d t\right)^{\frac{r+1}{r+\frac{p}{2}+1}} \\
& \left(\int_{Q_{R}} \phi^{k-\frac{2 r+p+2}{p}}\left|\frac{\partial \phi}{\partial t}\right|^{\frac{2 r+p+2}{p}} d \mu d t\right)^{\frac{p}{2 r+p+2}} \\
& \leq \int_{Q_{R}} g^{r+\frac{p}{2}+1} \phi^{k} d \mu d t+C \int_{Q_{R}} \phi^{k-\frac{r+3}{2}}\left|\frac{\partial \phi}{\partial t}\right|^{\frac{2 r+p+2}{p}} d \mu d t \\
& \leq \int_{Q_{R}} g^{r+\frac{p}{2}+1} \phi^{k} d \mu d t+C R(R-\rho)^{-\frac{2 r+p+2}{p}}\left|B\left(x_{0}, R\right)\right|,
\end{aligned}
$$

where $C$ is a constant depending on $M, p$ and $r$. By choosing $k \geq 2\left(r+\frac{p}{2}+1\right)$, and using Hölder's inequality and Young's inequality, we have

$$
\begin{aligned}
& \int_{Q_{R}} g^{\frac{p}{2}+r}\left[\phi^{k}+\phi^{k-2}\left|\nabla_{b} \phi\right|^{2}\right] d \mu d t \\
& \leq \int_{Q_{R}} g^{r+\frac{p}{2}+1} \phi^{k} d \mu d t+C R\left[1+(R-\rho)^{-(2 r+p+2)}\right]\left|B\left(x_{0}, R\right)\right|,
\end{aligned}
$$

where $C$ is a constant depending on $M, p$ and $r$. It follows from (4.17), (4.18) and (4.19) that

$$
\begin{aligned}
& \sup _{t \leq t_{0}} \int_{B\left(x_{0}, R\right)} g^{1+r} \phi^{k} d \mu+\int_{Q_{R}} g^{r+\frac{p}{2}-2}\left|\nabla_{b} g\right|^{2} \phi^{k} d \mu d t \leq C_{1} \int_{Q_{R}} g^{r+\frac{p}{2}+1} \phi^{k} d \mu d t \\
& +C_{1} R\left[1+(R-\rho)^{-(p+2 r+2)}+(R-\rho)^{-\frac{2 r+p+2}{p}}\right]\left|B\left(x_{0}, R\right)\right|,
\end{aligned}
$$

where $C_{1}$ is a constant depending on $M, N, p$ and $r$. We recall the following CR Sobolev inequality $([\mathrm{J}]$, [Lu, Theorem C] or [DLS, (1.1)])

$$
\left(\int_{B\left(x_{0}, R\right)} \omega^{q} d \mu\right)^{\frac{1}{q}} \leq c\left(\int_{B\left(x_{0}, R\right)}\left|\nabla_{b} \omega\right|^{p} d \mu\right)^{\frac{1}{p}},
$$

where $\omega \in C_{0}^{1}\left(B\left(x_{0}, R\right)\right)$ and $c$ is a constant, provided that $1 \leq p<Q=2 n+2$ and $\frac{1}{p}-\frac{1}{q}=\frac{1}{Q}$. In particular for $q=2$, we have

$$
\left(\int_{B\left(x_{0}, R\right)} \omega^{2} d \mu\right) \leq c\left(\int_{B\left(x_{0}, R\right)}\left|\nabla_{b} \omega\right|^{\frac{2(n+1)}{n+2}} d \mu\right)^{\frac{n+2}{n+1}}
$$


Applying this inequality to $\omega=g^{\frac{r+\frac{p}{2}+1}{2}} \psi^{\frac{k}{2}}$,

$$
\begin{aligned}
& \int_{B\left(x_{0}, R\right)} g^{r+\frac{p}{2}+1} \psi^{k} d \mu \\
& \leq c\left(\int_{B\left(x_{0}, R\right)}\left|\nabla_{b}\left(g^{\frac{r+\frac{p}{2}+1}{2}} \psi^{\frac{k}{2}}\right)\right|^{\frac{2 n+2}{n+2}} d \mu\right)^{\frac{n+2}{n+1}} \\
& =c\left(\int_{B\left(x_{0}, R\right)}\left|\frac{r+\frac{p}{2}+1}{2} g^{\frac{r+\frac{p}{2}-1}{2}} \psi^{\frac{k}{2}} \nabla_{b} g+\frac{k}{2} g^{\frac{r+\frac{p}{2}+1}{2}} \psi^{\frac{k-2}{2}} \nabla_{b} \psi\right|^{\frac{2 n+2}{n+2}} d \mu\right)^{\frac{n+2}{n+1}} \\
& \leq C\left(\int_{B\left(x_{0}, R\right)}\left|g^{\frac{r+\frac{p}{2}-1}{2}} \psi^{\frac{k}{2}} \nabla_{b} g\right|^{\frac{2 n+2}{n+2}} d \mu\right)^{\frac{n+2}{n+1}}+C\left(\int_{B\left(x_{0}, R\right)}\left|g^{\frac{r+\frac{p}{2}+1}{2}} \psi^{\frac{k-2}{2}} \nabla_{b} \psi\right|^{\frac{2 n+2}{n+2}} d \mu\right)^{\frac{n+2}{n+1}} \\
& \leq C_{2}\left(\int_{B\left(x_{0}, R\right)} g^{n+1} d \mu\right)^{\frac{1}{n+1}}\left(\int_{B\left(x_{0}, R\right)} \psi^{k} g^{r+\frac{p}{2}-2}\left|\nabla_{b} g\right|^{2} d \mu\right) \\
& +C_{2}\left(\int_{B\left(x_{0}, R\right)}\left|g^{\frac{r+\frac{p}{2}+1}{2}} \psi^{\frac{k-2}{2}} \nabla_{b} \psi\right|^{\frac{2 n+2}{n+2}} d \mu\right)^{\frac{n+2}{n+1}} \\
& =C_{2}|| g(\cdot, t)|| L^{n+1\left(B\left(x_{0}, R\right)\right)}\left(\int_{B\left(x_{0}, R\right)} \psi^{k} g^{r+\frac{p}{2}-2}\left|\nabla_{b} g\right|^{2} d \mu\right) \\
& +C_{2}\left(\int_{B\left(x_{0}, R\right)}\left|g^{\frac{r+\frac{p}{2}+1}{2}} \psi^{\frac{k-2}{2}} \nabla_{b} \psi\right|^{\frac{2 n+2}{n+2}} d \mu\right)^{\frac{n+2}{n+1}},
\end{aligned}
$$

where $C_{2}$ is a constant depending on $M, p$ and $r$. Multiply (4.23) by $\eta^{k}(t)$ and integrating on $\left[t_{0}-R, t_{0}\right]$,

$$
\begin{aligned}
& \int_{Q_{R}} g^{r+\frac{p}{2}+1} \phi^{k} d \mu d t \\
& \leq C_{2} \sup _{t_{0}-R<t \leq t_{0}}|| g(\cdot, t) \|_{L^{n+1}\left(B\left(x_{0}, R\right)\right)}\left(\int_{Q_{R}} \phi^{k} g^{r+\frac{p}{2}-2}\left|\nabla_{b} g\right|^{2} d \mu d t\right) \\
& +C_{2} \int_{t_{0}-R}^{t_{0}}\left(\int_{B\left(x_{0}, R\right)} g^{\left(r+\frac{p}{2}+1\right) \frac{n+1}{n+2}} \psi^{\frac{(k-2)(n+1)}{n+2}} \eta^{\frac{k(n+1)}{n+2}}\left|\nabla_{b} \psi\right|^{\frac{2 n+2}{n+2}} d \mu\right)^{\frac{n+2}{n+1}} d t .
\end{aligned}
$$

Set $\varepsilon_{1}=\frac{1}{2 C_{1} C_{2}}$. Suppose that

$$
\|g(\cdot, t)\|_{L^{n+1}\left(B\left(x_{0}, R\right)\right)} \leq \varepsilon_{1} .
$$


It follows from (4.20) and (4.24) that

$$
\begin{aligned}
& \sup _{t_{0}-R \leq t \leq t_{0}} \int_{B\left(x_{0}, R\right)} g^{1+r} \phi^{k} d \mu+\int_{Q_{R}} g^{r+\frac{p}{2}+1} \phi^{k} d \mu d t \\
& \leq C \int_{t_{0}-R}^{t_{0}}\left(\int_{B\left(x_{0}, R\right)} g^{\left(r+\frac{p}{2}+1\right) \frac{n+1}{n+2}} \psi^{\frac{(k-2)(n+1)}{n+2}} \eta^{\frac{k(n+1)}{n+2}}\left|\nabla_{b} \psi\right|^{\frac{2 n+2}{n+2}} d \mu\right)^{\frac{n+2}{n+1}} d t \\
& +C R\left[1+(R-\rho)^{-(p+2 r+2)}+(R-\rho)^{-\frac{2 r+p+2}{p}}\right]\left|B\left(x_{0}, R\right)\right| .
\end{aligned}
$$

From the definition of $\phi$, we obtain,

$$
\begin{aligned}
& \sup _{t_{0}-\rho \leq t \leq t_{0}} \int_{B\left(x_{0}, \rho\right)} g^{1+r} d \mu+\int_{Q_{\rho}} g^{r+\frac{p}{2}+1} d \mu d t \\
& \leq C(R-\rho)^{-2} \int_{t_{0}-R}^{t_{0}}\left(\int_{B\left(x_{0}, R\right)} g^{\frac{\left(r+\frac{p}{2}+1\right)(n+1)}{n+2}} d \mu\right)^{\frac{n+2}{n+1}} d t \\
& +C R\left[1+(R-\rho)^{-\frac{2 r+p+2}{p}}+(R-\rho)^{-(p+2 r+2)}\right]\left|B\left(x_{0}, R\right)\right| \\
& \leq C\left[1+\int_{t_{0}-R}^{t_{0}}\left(\int_{B\left(x_{0}, R\right)} f^{\frac{\left(r+\frac{p}{2}+1\right)(n+1)}{n+2}} d \mu\right)^{\frac{n+2}{n+1}} d t\right],
\end{aligned}
$$

where $C$ is a constant depending on $M, N, R, r, p$ and $\rho$. Let $d=\frac{(r+3)(n+1)}{n+2}+\frac{1}{n+2}(r+1)$. By using Hölder inequality,

$$
\begin{aligned}
& \int_{t_{0}-\rho}^{t_{0}}\left(\int_{B\left(x_{0}, \rho\right)} g^{\frac{\left(r+\frac{p}{2}+1\right)(n+1)}{n+2}+\frac{1}{n+2}(r+1)} d \mu\right)^{\frac{n+2}{n+1}} d t \\
& \leq \int_{t_{0}-\rho}^{t_{0}}\left[\int_{B\left(x_{0}, \rho\right)} g^{\left(r+\frac{p}{2}+1\right)} d \mu\left(\int_{B\left(x_{0}, \rho\right)} g^{r+1} d \mu\right)^{\frac{1}{n+1}}\right] d t \\
& \leq \sup _{t_{0}-\rho \leq t \leq t_{0}}\left(\int_{B\left(x_{0}, \rho\right)} g^{1+r} d \mu\right)^{\frac{1}{n+1}} \int_{Q_{\rho}} g^{\left(r+\frac{p}{2}+1\right)} d \mu d t .
\end{aligned}
$$

From (4.25) and (4.26), we obtain

$$
\begin{aligned}
& \int_{t_{0}-\rho}^{t_{0}}\left(\int_{B\left(x_{0}, \rho\right)} g^{\frac{\left(r+\frac{p}{2}+1\right)(n+1)}{n+2}+\frac{1}{n+2}(r+1)} d \mu\right)^{\frac{n+2}{n+1}} d t \\
& \leq C\left[1+\int_{t_{0}-R}^{t_{0}}\left(\int_{B\left(x_{0}, R\right)} g^{\frac{\left(r+\frac{p}{2}+1\right)(n+1)}{n+2}} d \mu\right)^{\frac{n+2}{n+1}} d t\right]^{\frac{n+2}{n+1}},
\end{aligned}
$$

where $C$ is a constant depending on $M, N, R, r, p$ and $\rho$. We set $\theta=1+\frac{1}{n+1}$ and for $s \in N$, $R_{s}=\left(1+2^{-s}\right) \frac{R_{0}}{2}$. Define $r_{s}=\left(\frac{2 n-p+4}{2}\right) \theta^{s}-1$, and $a_{s}=\left(r_{s}+\frac{p}{2}+1\right) \frac{n+1}{n+2}$. From the definitions 
of $r_{s}, a_{s}$, we have $a_{s+1}=\left(r_{s}+\frac{p}{2}+1\right) \frac{n+1}{n+2}+\left(r_{s}+1\right) \frac{1}{n+2}$. If $\rho=R_{s+1}, R=R_{s}$ and $r=r_{s}$ in (4.27), we have

$$
\begin{aligned}
& \int_{t_{0}-R_{s+1}}^{t_{0}}\left(\int_{B\left(x_{0}, R_{s+1}\right)} g^{a_{s+1}} d \mu\right)^{\frac{n+2}{n+1}} d t \\
& \leq C_{s}\left[1+\int_{t_{0}-R_{s}}^{t_{0}}\left(\int_{B\left(x_{0}, R_{s}\right)} g^{a_{s}} d \mu\right)^{\frac{n+2}{n+1}} d t\right]^{\frac{n+2}{n+1}},
\end{aligned}
$$

where $C_{s}$ is a constant depending on $M, N$ and $s$. Since $a_{s} \rightarrow \infty$ when $s \rightarrow \infty$, by interating (4.28), we have for any $q \geq 1$

$$
\|g\|_{L^{q}\left(\left[t_{0}-\frac{R_{0}}{2}, t_{0}\right] \times B\left(x_{0}, \frac{R_{0}}{2}\right)\right)} \leq C
$$

where the constant $C$ depends on $M, N, q$ and $\|g(\cdot, t)\|_{L^{a_{0}(M)}}$. Since $a_{0}=n+1$ and by hypothesis $\sup _{0 \leq t \leq T^{\prime}}\|g(\cdot, t)\|_{L^{n+1}(M)} \leq \varepsilon_{1}$, then $C$ depends only on $M, N$ and $q$. Following the same steps as in proof of (4.29) where we take $\psi^{k}(x)$ instead of $\phi^{k}(x, t)$ and we integrate on $[0, t]$ (for $t \in[0,1]$ ) instead of $\left[t_{0}-R, t_{0}\right]$, we can obtain the following inequality:

$$
\|g\|_{L^{q}\left([0,1] \times B\left(x_{0}, \frac{R_{0}}{2}\right)\right)} \leq C
$$

where $C$ depends on $M, N, q$ and $K$ which is any positive constant such that $\left\|\nabla u_{0}\right\|_{L^{\infty}(M)} \leq$ $K$. Since $M$ is compact, we know that (4.2) is true by using (4.29) and (4.30). To prove (4.3), we proceed as in (4.30) where we integrate on $[0, t]$ with $t \in\left[0, T^{\prime}\right)$.

(ii) Note that as in $(i)$, we assume

$$
\sup _{0 \leq t<T^{\prime}}\|g(t, \cdot)\|_{L^{n+1}(M)} \leq \varepsilon_{1}
$$

in order to get the control of the term $\int_{Q_{R}} g^{r+\frac{p}{2}+1} \phi^{k} d \mu d t$ as in (4.24). However, if the sectional curvature of $\left(N, g_{i j}\right)$ is nonpositive

$$
K^{N} \leq 0
$$


it follows from (3.4) that we do not need to estimate this term any more. Then we have the estimates (4.2) and (4.3) without assumption (4.1) if the sectional curvature of $\left(N, g_{i j}\right)$ is nonpositive.

Lemma 4.2. Let $\left(M^{2 n+1}, J, \theta\right)$ be a closed Sasakian manifold and $(N, g)$ be a compact Riemannian manifold. Let $u_{0} \in C^{2, \alpha}(M, N), 0<\alpha<1,\left\|\nabla u_{0}\right\|_{L^{\infty}(M)} \leq K$ and $\varphi_{\delta}$ is the solution of the regularized equation (1.5).

(i) There exists $\varepsilon_{1}>0$ depending on $K, M, N$ such that if

$$
\sup _{0 \leq t<T^{\prime}}\|g(t, \cdot)\|_{L^{n+1}(M)} \leq \varepsilon_{1}
$$

then

$$
\|g\|_{L^{\infty}\left(\left[0, T^{\prime}\right) \times M\right)} \leq C
$$

where $C$ is a constant depending on $K, M$ and $N$.

(ii) In addition, if the sectional curvature of $\left(N, g_{i j}\right)$ is nonpositive

$$
K^{N} \leq 0
$$

then (4.32) holds without the smallness assumption (4.31). 
Proof. ( $i$ ) Let $\phi(x, t)=\psi(x) \eta(t)$ and $Q_{R}$ is as in the proof of Lemma 4.1, Multiplying inequality (3.3) by $g^{r} \phi^{2}, r \geq 0$ and similar to (4.4), we have

$$
\begin{aligned}
& \frac{1}{1+r} \sup _{t \leq t_{0}} \int_{B\left(x_{0}, R\right)} g^{1+r} \phi^{2} d \mu+\frac{r}{2} \int_{Q_{R}} f_{\delta}^{\frac{p-2}{2}} g^{r-1}\left|\nabla_{b} g\right|^{2} \phi^{2} d \mu d t \\
& +(p-2)\left(\frac{3 r}{4}-1\right) \int_{Q_{R}} f_{\delta}^{\frac{p-4}{2}} g^{r-1}\left\langle\nabla_{b} f_{\delta}, \nabla_{b} \varphi^{l}\right\rangle^{2} \phi^{2} d \mu d t \\
& -(p-2) r^{2} \varepsilon \int_{Q_{R}} f_{\delta}^{\frac{p-4}{2}} g^{r-2} e_{0}\left\langle\nabla_{b} \varphi^{l}, \nabla_{b} f_{\delta}\right\rangle^{2} \phi^{2} d \mu d t \\
& +\frac{p-2}{2} \int_{Q_{R}} f_{\delta}^{\frac{p-4}{2}} g^{r} \phi^{2}\left|\nabla_{b} f_{\delta}\right|^{2} d \mu d t+2 \int_{Q_{R}} f_{\delta}^{\frac{p-2}{2}}\left|\nabla_{b}^{2} \varphi^{l}\right|^{2} g^{r} \phi^{2} d \mu d t \\
& +\frac{7 \varepsilon}{4} \int_{Q_{R}} f_{\delta}^{\frac{p-2}{2}}\left|\nabla_{b} \varphi_{0}^{l}\right|^{2} g^{r} \phi^{2} d \mu d t-(p-2) r^{2} \varepsilon^{3} \int_{Q_{R}} f_{\delta}^{\frac{p-2}{2}} g^{r-2} e_{0}^{2}\left|\nabla_{b} \varphi_{0}^{l}\right|^{2} \phi^{2} d \mu d t \\
& -4(p-2) r \varepsilon^{2} \int_{Q_{R}} f_{\delta}^{\frac{p-2}{2}}\left|\nabla_{b} \varphi_{0}^{l}\right|^{2} e_{0} g^{r-1} \phi^{2} d \mu d t \\
& \leq \frac{2}{1+r} \int_{Q_{R}} g^{1+r} \phi\left|\frac{\partial \phi}{\partial t}\right| d \mu d t+4(p-2) \varepsilon \int_{Q_{R}} f_{\delta}^{\frac{p-2}{2}} g^{r} e_{0}\left|\nabla_{b} \phi\right|^{2} d \mu d t \\
& +\frac{8}{r} \int_{Q_{R}} f_{\delta}^{\frac{p-2}{2}} g^{1+r}\left|\nabla_{b} \phi\right|^{2} d \mu d t+(p-2) \int_{Q_{R}} f_{\delta}^{\frac{p-2}{2}} g^{1+r}\left|\nabla_{b} \phi\right|^{2} d \mu d t \\
& +\frac{16}{\varepsilon} \int_{Q_{R}} f_{\delta}^{\frac{p}{2}} g^{r} \phi^{2} d \mu d t+C \int_{Q_{R}}\left[f_{\delta}^{\frac{p}{2}}+f_{\delta}^{\frac{p+2}{2}}+\varepsilon f_{\delta}^{\frac{p}{2}} e_{0}\right] g^{r} \phi^{2} d \mu d t .
\end{aligned}
$$

For any fixed $t$, we choose $\varepsilon$ small enough so that

$$
\begin{aligned}
& \frac{1}{1+r} \sup _{t \leq t_{0}} \int_{B\left(x_{0}, R\right)} g^{1+r} \phi^{2} d \mu+\frac{r}{2} \int_{Q_{R}} g^{r+\frac{p}{2}-2}\left|\nabla_{b} g\right|^{2} \phi^{2} d \mu d t \\
& \leq C \int_{Q_{R}} g^{1+r} \phi\left|\frac{\partial \phi}{\partial t}\right| d \mu d t+C \int_{Q_{R}} g^{r+\frac{p}{2}}\left|\nabla_{b} \phi\right|^{2} d \mu d t+C \int_{Q_{R}}\left(g^{r+\frac{p}{2}}+g^{r+\frac{p}{2}+1}\right) \phi^{2} d \mu d t
\end{aligned}
$$

where $C$ is a positive constant depending on $M, N, r$ and $p$. Since

$$
\int_{Q_{R}} g^{r+\frac{p}{2}-2}\left|\nabla_{b} g\right|^{2} \phi^{2} d \mu d t \geq \frac{8}{(p+2 r)^{2}} \int_{Q_{R}}\left|\nabla_{b}\left(g^{\frac{r}{2}+\frac{p}{4}} \phi\right)\right|^{2} d \mu d t-\frac{16}{(p+2 r)^{2}} \int_{Q_{R}} g^{r+\frac{p}{2}}\left|\nabla_{b} \phi\right|^{2} d \mu d t
$$


So

$$
\begin{aligned}
& \frac{1}{1+r} \sup _{t \leq t_{0}} \int_{B\left(x_{0}, R\right)} g^{1+r} \phi^{2} d \mu+\frac{4 r}{(p+2 r)^{2}} \int_{Q_{R}}\left|\nabla_{b}\left(g^{\frac{r}{2}+\frac{p}{4}} \phi\right)\right|^{2} d \mu d t \\
& \leq C \int_{Q_{R}} g^{1+r} \phi\left|\frac{\partial \phi}{\partial t}\right| d \mu d t+\left(C+\frac{8 r}{(p+2 r)^{2}}\right) \int_{Q_{R}} g^{r+\frac{p}{2}}\left|\nabla_{b} \phi\right|^{2} d \mu d t \\
& +C \int_{Q_{R}}\left(g^{r+\frac{p}{2}}+g^{r+\frac{p}{2}+1}\right) \phi^{2} d \mu d t
\end{aligned}
$$

From the definition of $\phi$,

$$
\begin{aligned}
& \sup _{t \leq t_{0}} \int_{B\left(x_{0}, R\right)} g^{1+r} \phi^{2} d \mu+\int_{Q_{R}}\left|\nabla_{b}\left(g^{\frac{r+2}{2}} \phi\right)\right|^{2} \mu d t \\
& \leq C\left[(1+r) \int_{Q_{R}} g^{\frac{p}{2}+r} d \mu d t+(1+r) \int_{Q_{R}} g^{1+\frac{p}{2}+r} \phi^{2} d \mu d t\right. \\
& \left.+(R-\rho)^{-1} \int_{Q_{R}} g^{1+r} d \mu d t+(R-\rho)^{-2} \int_{Q_{R}} g^{\frac{p}{2}+r} d \mu d t\right]
\end{aligned}
$$

where $C$ is a constant depending on $M, N$. Let $q=\left(1+\frac{1}{n+1}\right) r+\left(\frac{p}{2}+\frac{1}{n+1}\right)$. By using Hölder inequality,

$$
\begin{aligned}
& \int_{Q_{\rho}} g^{q} d \mu d t \\
& \leq \sup _{t \leq t_{0}}\left(\int_{B\left(x_{0}, R\right)} g^{1+r} \phi^{2} d \mu\right)^{\frac{1}{n+1}}\left(\int_{t_{0}-R}^{t_{0}}\left(\int_{B\left(x_{0}, R\right)}\left(g^{\frac{r}{2}+\frac{p}{4}} \phi\right)^{\frac{2(n+1)}{n}} d \mu\right)^{\frac{n}{n+1}} d t\right) .
\end{aligned}
$$

From the CR Sobolev inequality (4.21) again (with $p=2$ ), we have

$$
\left(\int_{B\left(x_{0}, R\right)} V^{\frac{2(n+1)}{n}} d \mu\right)^{\frac{n}{2 n+2}} \leq c\left(\int_{B\left(x_{0}, R\right)}\left|\nabla_{b} V\right|^{2} d \mu\right)^{\frac{1}{2}}
$$

for all $V \in C_{0}^{1}\left(B\left(x_{0}, R\right)\right)$. Let $V=g^{\frac{r}{2}+\frac{p}{4}} \phi$,

$$
\left(\int_{B\left(x_{0}, R\right)}\left(g^{\frac{r}{2}+\frac{p}{4}} \phi\right)^{\frac{2(n+1)}{n}} d \mu\right)^{\frac{n}{n+1}} \leq c \int_{B\left(x_{0}, R\right)}\left|\nabla_{b}\left(g^{\frac{r}{2}+\frac{p}{4}} \phi\right)\right|^{2} d \mu .
$$

It follows from (4.36) and (4.38) that

$$
\int_{Q_{\rho}} g^{q} d \mu d t \leq c \sup _{t \leq t_{0}}\left(\int_{B\left(x_{0}, R\right)} g^{1+r} \phi^{2} d \mu\right)^{\frac{1}{n+1}}\left(\int_{Q_{R}}\left|\nabla_{b}\left(g^{\frac{r}{2}+\frac{p}{4}} \phi\right)\right|^{2} \mu d t\right) .
$$


and from (4.35) and (4.39) that

$$
\begin{aligned}
& \int_{Q_{\rho}} g^{q} d \mu d t \leq C\left[\left[(1+r)+(R-\rho)^{-2}\right] \int_{Q_{R}} g^{\frac{p}{2}+r} d \mu d t\right. \\
& \left.+(R-\rho)^{-1} \int_{Q_{R}} g^{1+r} d \mu d t+(1+r) \int_{Q_{R}} f^{\frac{p}{2}+r+1} d \mu d t\right]^{\frac{n+2}{n+1}} .
\end{aligned}
$$

By using Hölder inequality and Young's inequality in (4.40),

$$
\begin{aligned}
& \int_{Q_{\rho}} g^{q} d \mu d t \leq C\left\{\left[(1+r)^{\frac{2 r+p+2}{2 r+p}}+(R-\rho)^{\frac{-4 r-2 p-4}{2 r+p}}\right.\right. \\
& \left.\left.+(R-\rho)^{\frac{-2 r-p-2}{2 r+2}}\right] \int_{Q_{R}} g^{\frac{p}{2}+r+1} d \mu d t+\left|Q_{R}\right|\right\}^{\frac{n+2}{n+1}},
\end{aligned}
$$

where $C$ depends on $M, N$ and $p$. Now we use a Moser iteration process to obtain a uniform estimate. Let $R_{s}=\left(1+2^{-s}\right) \frac{R_{0}}{2}$ and $\theta=1+\frac{1}{n+1}$. Define $r_{s}$ and $a_{s}$ by

$$
r_{s}=\theta^{s}+n \quad \text { and } \quad a_{s}=r_{s}+\frac{p}{2}+1
$$

so that

$$
a_{s+1}=\theta r_{s}+\frac{p}{2}+\frac{1}{n+1}
$$

We apply (4.41) with $\rho=R_{s+1}, R=R_{s}$ and $r=r_{s}$ to find

$$
\int_{Q_{R_{s+1}}} g^{a_{s+1}} d \mu d t \leq C\left\{\int_{Q_{R_{s}}} g^{a_{s}} d \mu d t+C\right\}^{\frac{n+2}{n+1}}
$$

where $C$ is a positive constant depending only on $M, N$. By Moser iteration process, we have

$$
\|g\|_{L^{\infty}\left(Q_{\frac{R_{0}}{2}}\right)} \leq C\left\{\int_{Q_{R_{0}}} g^{a_{0}} d \mu d t+C\right\} .
$$

Now we suppose that

$$
\sup _{0 \leq t \leq T^{\prime}}\|g(\cdot, t)\|_{L^{n+1}(M)} \leq \varepsilon_{1}
$$

with $\varepsilon_{1}=\varepsilon$, where $\varepsilon$ is the constant in Lemma 3.3 corresponding to $q=a_{0}=n+\frac{p}{2}+2$. By Lemma 4.1, the right hand side of (4.42) is bounded by a constant depending on $K, M, N$. Since $M$ is compact, we have

$$
\|g\|_{L^{\infty}\left(\left[0, T^{\prime}\right) \times M\right)} \leq C .
$$


(ii) The same proof without the smallness assumption if the sectional curvature of $\left(N, g_{i j}\right)$ is nonpositive, we have the estimate (4.32).

Now we are ready to the proof of Theorem 1.1 :

Proof. (i) First, let us show that

$$
\sup _{0 \leq t \leq T^{\prime}}\|g(\cdot, t)\|_{L^{n+1}(M)} \leq \varepsilon_{1}
$$

where $g=f_{\delta}+\varepsilon e_{0}(\varphi)$. To this end, we set

$$
T^{*}=\sup \left\{\tilde{T} \in\left[0, T^{\prime}\right): \sup _{0 \leq t \leq \tilde{T}}\|g(\cdot, t)\|_{L^{n+1}(M)} \leq \varepsilon_{1}\right\} .
$$

We want to prove that $T^{*}=T^{\prime}$. If $T^{*}<T^{\prime}$, we have

$$
\sup _{0 \leq t \leq T^{*}}\|g(\cdot, t)\|_{L^{n+1}(M)} \leq \varepsilon_{1}
$$

So from Lemma 4.2, we have

$$
\|g(\cdot, t)\|_{L^{\infty}\left(\left[0, T^{*}\right] \times M\right)} \leq C .
$$

From this inequality, we have

$$
f_{\delta} \leq C \quad \text { and } \quad \varepsilon e_{0} \leq C
$$

Take $\varepsilon$ small enough, we have

$$
\varepsilon e_{0} \leq \frac{\varepsilon_{0}}{2}
$$

where $\varepsilon_{0}$ satisfying

$$
E_{p}\left(u_{0}\right)=\frac{1}{p} \int_{M}\left|\nabla_{b} u_{0}\right|^{p} d \mu \leq \varepsilon_{0} .
$$

On the other hand, we have for all $t \in\left[0, T^{*}\right]$,

$$
\|g(\cdot, t)\|_{L^{n+1}(M)}^{n+1} \leq\|g\|_{L^{\infty}\left(\left[0, T^{*}\right] \times M\right)}^{n+1-\frac{p}{2}} \|\left. g(\cdot, t)\right|_{L^{\frac{p}{2}}(M)} ^{\frac{p}{2}} .
$$


Since $(x+y)^{p} \leq C_{p}\left(x^{p}+y^{p}\right)$ for all $p>1$ and we can choose $\delta<\frac{\varepsilon_{0}}{2}$, we have

$$
\|g\|_{L^{\frac{p}{2}(M)}}^{\frac{p}{2}}=\int_{M}\left[\left|\nabla_{b} \varphi_{\delta}\right|^{2}+\delta+\varepsilon e_{0}\right]^{\frac{p}{2}} d v \leq C_{p} \int_{M}\left[\left|\nabla_{b} u_{0}\right|^{p} d v+\varepsilon_{0}^{\frac{p}{2}} \operatorname{Vol}(M) \leq C_{1} \varepsilon_{0} .\right.
$$

From (4.43) and (4.44), we have

$$
\sup _{0 \leq t \leq T^{*}}\|g(\cdot, t)\|_{L^{n+1}(M)}^{n+1} \leq C^{n-1} C_{1} \varepsilon_{0} .
$$

We can take $\varepsilon_{0}$ such that $\left[C^{n-1} C_{1} \varepsilon_{0}\right]^{\frac{1}{n+1}}<\frac{\varepsilon_{1}}{2}$, so we have

$$
\sup _{0 \leq t \leq T^{*}}\|g(\cdot, t)\|_{L^{n+1}(M)} \leq\left[C^{n} C_{1} \varepsilon_{0}\right]^{\frac{1}{n+1}} \leq \frac{\varepsilon_{1}}{2} .
$$

Since $f$ is continuous, we see that there exists $h>0$ such that

$$
\sup _{0 \leq t \leq T^{*}+h}\|g(\cdot, t)\|_{L^{n+1}(M)} \leq \frac{3 \varepsilon_{1}}{4} \leq \varepsilon_{1},
$$

so contradicting the definition of $T^{*}$. Then $T^{*}=T^{\prime}$ and $\sup _{0 \leq t \leq T^{*}+h}\|g(\cdot, t)\|_{L^{n+1}(M)} \leq \varepsilon_{1}$. By Lemma 4.2, we have

$$
\|g(\cdot, t)\|_{L^{\infty}\left(\left[0, T^{\prime}\right) \times M\right)} \leq C .
$$

That is

$$
\left\|\nabla_{b} \varphi_{\delta}\right\|_{L^{\infty}\left(\left[0, T^{\prime}\right) \times M\right)} \leq C \quad \text { and } \quad\left\|\mathbf{T} \varphi_{\delta}\right\|_{L^{\infty}\left(\left[0, T^{\prime}\right) \times M\right)} \leq C,
$$

where $C$ is a constant depending on $K, M$ and $N$.

(ii) The same proof as in (i) except we do not need the smallness assumption (1.6) due to $K_{N} \leq 0$.

(iii) By integration by parts, we compute as in [CC1, Lemma 3.1.], one has

$$
\frac{d}{d s} E_{p, \delta}\left(\varphi_{\delta}(\cdot, s)\right)=-\int_{M}\left|\partial_{s} \varphi_{\delta}(\cdot, s)\right|^{2} d \mu .
$$

Integrating the above equality over $[0, t]$ gives

$$
\int_{0}^{t} \int_{M}\left|\partial_{s} \varphi_{\delta}\right|^{2}(x, s) d \mu d s+E_{p, \delta}\left(\varphi_{\delta}(\cdot, t)\right)=E_{p, \delta}\left(u_{0}\right) \leq E_{p, 1}\left(u_{0}\right), \quad \forall t \in\left[0, T_{\delta}\right) .
$$


Now we are ready to prove the global existence and asymptotic convergence of the $p$ pseudoharmonic map heat flow. We will give the proof of Theorem 1.3 which is based on [FR2 and [CCW]. The proof of Theorem 1.2 is the similar to the proof of Theorem 1.3 .

The proof of Theorem 1.3 :

Proof. We first observe that the proof is standard once we have the estimate (1.7) plus CR divergence theorem and Green's identity as in [CCW]. We will sketch the proof and refer to the last section of [FR2] for more details.

(i) Global Existence : Firstly, it follows from [HS], [D], [DF] and [Ch] that there exist constants $\beta \in(0,1)$ and $C$ depending only on $M, N, p$ such that

$$
\left\|\varphi_{\delta}\right\|_{C^{\beta}\left(M \times\left[0, T_{\delta}\right), N\right)}+\left\|\nabla_{b} \varphi_{\delta}\right\|_{C^{\beta}\left(M \times\left[0, T_{\delta}\right), N\right)} \leq C .
$$

This and the theory of parabolic equations ([LSU] $)$ imply

$$
\sup _{0 \leq t<T_{\delta}}\left(\left\|\varphi_{\delta}(\cdot, t)\right\|_{C^{2+\alpha}(M, N)}+\left\|\partial_{t} \varphi_{\delta}(\cdot, t)\right\|_{C^{\alpha}(M, N)} \leq C_{\delta}\right.
$$

for $0<\alpha<1$. It is clear that the maximal time will be infinite

$$
T_{\delta}=\infty
$$

Moreover, by the energy inequality (1.8), there exist a sequence $\delta_{k} \rightarrow 0$ and $\varphi \in C^{\beta}(M \times$ $[0, \infty), N)$ and $\nabla_{b} \varphi \in C^{\beta}(M \times[0, \infty), N)$ such that

$$
\left\{\begin{array}{l}
(i)\left\|\nabla_{b} \varphi\right\|_{C^{\beta}(M \times[0, \infty), N)} \leq C_{1}, \\
(i i) \partial_{t} \varphi \in L^{2}(M \times[0, \infty)),
\end{array}\right.
$$

and

$$
\left\{\begin{aligned}
\varphi_{\delta_{k}} & \rightarrow \varphi \text { in } C_{l o c}^{\beta^{\prime}}(M \times[0, \infty), N) \text { for all } \beta^{\prime}<\beta, \\
\nabla_{b} \varphi_{\delta_{k}} & \rightarrow \nabla_{b} \varphi \quad \text { in } C_{l o c}^{\beta^{\prime}}(M \times[0, \infty), N) \text { for all } \beta^{\prime}<\beta, \\
\partial_{t} \varphi_{\delta_{k}} & \rightarrow \partial_{t} \varphi \quad \text { weakly in } L^{2}(M \times[0, \infty)) .
\end{aligned}\right.
$$


Now by multiplying $\psi \in C_{0}^{\infty}(M \times[0, \infty))$ for (1.5) and integrating on $M \times[0, \infty)$, we have

$$
\begin{aligned}
& \int_{0}^{\infty} \int_{M} \partial_{t} \varphi \psi d \mu d t+\int_{0}^{\infty} \int_{M}\left|\nabla_{b} \varphi\right|^{p-2} \nabla_{b} \varphi \nabla_{b} \psi d \mu d t \\
& =2 \int_{0}^{\infty} \int_{M}\left|\nabla_{b} \varphi\right|^{p-2} h^{\alpha \bar{\beta}} \tilde{\Gamma}_{i j}^{k} \varphi_{\alpha}^{i} \varphi_{\bar{\beta}}^{j} \psi d \mu d t,
\end{aligned}
$$

as $\delta_{k} \rightarrow 0$. hence $\varphi$ is a weak solution of (1.4). The uniqueness is also standard. We refer to the last section of [FR2] for more details.

(ii) Asymptotic Convergence : Since $\partial_{t} \varphi \in L^{2}(M \times[0, \infty))$, it follows from (4.46) that for almost $t>0$ and $\phi \in C^{\infty}(M)$

$$
\int_{M} \partial_{t} \varphi \phi d \mu+\int_{M}\left|\nabla_{b} \varphi\right|^{p-2} \nabla_{b} \varphi \nabla_{b} \phi d \mu=2 \int_{M}\left|\nabla_{b} \varphi\right|^{p-2} h^{\alpha \bar{\beta}} \tilde{\Gamma}_{i j}^{k} \varphi_{\alpha}^{i} \varphi_{\bar{\beta}}^{j} \phi d \mu
$$

and then there exists a sequence $t_{k} \rightarrow \infty$ such that

$$
\left\|\partial_{t} \varphi\left(t_{k}, \cdot\right)\right\|_{L^{2}(M)} \rightarrow 0
$$

Furthermore, we have

$$
\left\|\varphi\left(t_{k}, \cdot\right)\right\|_{C^{1+\beta}(M, N)} \leq C
$$

and then

$$
\varphi\left(t_{k}, \cdot\right) \rightarrow \varphi_{\infty}(\cdot) \text { in } C^{1+\beta^{\prime}}(M, N) \text { for all } \beta^{\prime}<\beta .
$$

Moreover $\varphi_{\infty}$ is a weakly $p$-harmonic map and $\varphi_{\infty} \in C^{1+\beta}(M, N)$ with

$$
E_{p}\left(\varphi_{\infty}\right) \leq E_{p}\left(u_{0}\right)
$$

In addition if we choose $\overline{\varepsilon_{0}}>0$ such that $E_{p}\left(u_{0}\right) \leq \overline{\varepsilon_{0}}$, then for a fixed $q>Q=2 n+2$

$$
\int_{M}\left|\nabla_{b} \varphi_{\infty}\right|^{q} d \mu \leq p C^{q-p} \overline{\varepsilon_{0}}
$$

and for $N \subset \mathbf{R}^{l}$

$$
\left\|\varphi_{\infty}\right\|_{S^{1, q}\left(M, \mathbf{R}^{l}\right)} \leq C \overline{\varepsilon_{0}}
$$


Here we apply Poincare inequality by changing the origin in $\mathbf{R}^{l}$ such that $\int_{M} \varphi_{\infty} d \mu=0$. It follows from CR Sobolev embedding theorem ([FS, Theorem 21.1.], [JL]) that

$$
\left\|\varphi_{\infty}\right\|_{C^{\frac{1}{2}\left(1-\frac{Q}{q}\right)}\left(M, \mathbf{R}^{l}\right)} \leq C \overline{\varepsilon_{0}} .
$$

Thus if we choose $\overline{\varepsilon_{0}}$ small enough, $\varphi_{\infty}(M)$ is contained in a convex geodesic ball $U$ of $N$. By following the first name author's previous result as in [CC2, Lemma 4.2.] (or [Gor]), we consider the composite function $G:=F \circ \varphi$ with a smooth function $F$ defined on $U$. In particular, let us choose $F(y)=\sum_{j}\left(y_{j}\right)^{2}$, where $\left\{y_{j}\right\}_{j}$ is the coordinate system of $U$. Thus

$$
\begin{aligned}
& \operatorname{div}_{b}\left(\left|\nabla_{b} \varphi_{\infty}\right|^{p-2} \nabla_{b} G\right) \\
& =\sum_{k} F_{k}\left[\operatorname{div}_{b}\left(\left|\nabla_{b} \varphi\right|^{p-2} \nabla_{b} \varphi^{k}\right)+2\left|\nabla_{b} \varphi\right|^{p-2} h^{\alpha \bar{\beta}} \tilde{\Gamma}_{i j}^{k} \varphi_{\alpha}^{i} \varphi_{\bar{\beta}}^{j}\right] \\
& \quad+\left|\nabla_{b} \varphi_{\infty}\right|^{p-2} \sum_{i, j} \sum_{\alpha} F_{i j}\left(\varphi_{\infty}^{i}\right)_{\alpha}\left(\varphi_{\infty}^{j}\right)_{\bar{\alpha}} \\
& =\sum_{k} F_{k}\left[\operatorname{div}_{b}\left(\left|\nabla_{b} \varphi\right|^{p-2} \nabla_{b} \varphi^{k}\right)+2\left|\nabla_{b} \varphi\right|^{p-2} h^{\alpha \bar{\beta}} \tilde{\Gamma}_{i j}^{k} \varphi_{\alpha}^{i} \varphi_{\bar{\beta}}^{j}\right]+\left|\nabla_{b} \varphi_{\infty}\right|^{p} .
\end{aligned}
$$

Since $\varphi_{\infty}$ is a weakly $p$-harmonic map, we obtain by integrating both sides

$$
\int_{M}\left|\nabla_{b} \varphi_{\infty}\right|^{p} d \mu=0
$$

and we deduce that $\nabla_{b} \varphi_{\infty}=0$. This completes the proof of Theorem 1.3.

\section{REFERENCES}

[BDU] E. Barletta, S. Dragomir and H. Urakawa, Pseudoharmonic Maps from Nondegenerate CR Manifolds to Riemannian Manifolds, IUMJ Vol. 50 (2001), 719-746.

[CC1] S.-C. Chang and T.-H. Chang, On the existence of pseudoharmonic maps form pseudohermtian manifolds into Riemannian manifolds with nonpositive sectional curavature, Asian J. Math. 17 (2013), $1-16$.

[CC2] S.-C. Chang and T.-H. Chang, Finite-time blow up for the heat flow of pseudoharmonic maps, Indiana Uni. Math. J. 64 (2015), 441-470.

[CCW] S.-C. Chang, C.-W. Chen and C.-T. Wu, On the CR Analogue of Reilly Formula and Yau Eigenvalue Conjecture, arXiv:1503.07639.

[CD] Y. Chen and W.-Y. Ding, Blow-up and global existence for heat flows of harmonic maps, Invent. Math. 99 (1990), 567-578. 
[CDRY] T. Chong, Y. Dong, Y. Ren and G. Yang, On Harmonic and Pseudoharmonic Maps from Strictly Pseudoconvex CR Manifolds, arXiv: 1505.02170v1.

[Ch] H.J. Choe, Hőlder regularity for the gradients of solutions of certain parabolic equations, Comm. Partial Differential Equations 16 (1991), 1709-1732.

[CHH] Y. Chen, M.-C. Hong and N. Hungerbuhler, Heat flow of $p$-harmonic maps with values into spheres, Math. Z. 215 (1994), 25-35.

[CS] Y. Chen and M. Struwe, Existence and partial regularity results for the heat flow for harmonic maps, Math. Z. (1989) 201, 83-103.

[D] E. Dibenedetto, Degenerate parabolic equations, Universitext, Springer, Berlin, 1993.

[DF] E. Dibenedetto and A. Friedman, Hőlder estimates for nonlinear degenerate parabolic systems, J. Reine. Angew. Math. 357 (1984), 1-22.

[DT] S. Dragomir and G. Tomassini, Differential Geometry and Analysis on CR Manifolds, Progress in Mathematics, 246. Birkhauser Boston, Inc., Boston, MA, 2006. xvi+487 pp.

[ES] J. Eells and J. H. Sampson, Harmonic mappings of Riemannian manifolds, Amer. J. Math., 86(1964), 109-160.

[DLS] Y. Dong, G. Lu and L. Sun, Global Poincare Inequalities on the Heisenberg Group and Applications, Acta Mathematica Sinica, English Series Apr., Vol. 23, No. 4 (2007), pp. 735-744.

[F] G. B. Folland, Subelliptic estimates and function spaces on nilpotent Lie groups. ARKIV FÖR MATEMATIK, Volume 13, Number 1-2 (1975), 161-207.

[FR1] A. Fardoun and R. Regbaoui, Heat flow for $p$-harmonic maps between compact Riemannian manifolds, Indiana Uni. Math. J. (2002), 1305-1320.

[FR2] A. Fardoun and R. Regbaoui, Heat flow for $p$-harmonic maps with small intial data, Calc. Var. 16(2003), 1-16.

[FS] G. B. Folland and E. M. Stein, Estimates for the $\bar{\partial}_{b}$ Complex and Analysis on the Heisenberg Group, Comm. Pure Appl. Math., 27 (1974), 429-522.

[G] A. Greenleaf, The first eigenvalue of a sublaplacian on a pseudohermitian manifold, Comm. Part. Diff. Equ. 10(2) No.3 (1985), 191-217.

[Gor] W. B. Gordon, Convex functions and harmonic maps, Proc. Amer. Math. Soc. 33 (1972), 433-437.

[H1] N. Hungerbuhler, Global weak solutions of the $p$-harmonic flow into homogeneous space, Indiana Univ. Math. J 45/1 (1996), 275-288.

[H2] N. Hungerbuhler, m-Harmonic flow, Ann. Sc. Norm. Super. Pisa IV. Ser. 24, No.4 (1997), 593-631. 
[HL] R. Hardt and H.-F. Lin, Mappings minimizing the $L^{p}$-Norm of the Gradient, Communications on Pure and Applied Mathematics, Vol. XL (1987), 555-588.

[HS] P. Hájlasz and P. Strzelecki, Subelliptic p-harmonic maps into spheres and the ghost of Hardy spaces, Math. Ann. 312 (1998), 341-362.

[J] D. Jerison, The Poincare inequality for vector fields satisfying Hörmander's condition, Duke Math. J., 53 (1986), 503-523.

[JL] D. Jerison and J. M. Lee, The Yamabe problem on CR manifolds, J. Differential Geom. Volume 25, Number 2 (1987), 167-197.

$[\mathrm{K}] \quad$ B. Kim, Poincaré inequality and the uniqueness of solutions for the heat equation associated with subelliptic diffusion operators, arXiv:1305.0508,

[L] J. M. Lee, Pseudo-Einstein Structure on CR Manifolds, Amer. J. Math. 110 (1988), 157-178.

[LSU] O. A. Ladyzenskaya,V. A. Solonnikov and N. N. Ural'ceva, Linear and quasilinear equations of parabolic type, AMS Providence R.I., 1968.

[Lu] G. Lu, Weighted Poincare and Sobolev inequalities for vector fields satisfying Hörmander's condition and applications. Revista Mat. Iberoamericana, 8 (1992), 367-439.

[M] Jürgen Moser. A Harnack Inequality for Parabolic Differential Equations. Communications on pure and applied mathematics, vol. 17, 101-134 (1964).

[XZ] C.-J. Xu and C. Zuily, Higher interior regularity for quasilinear subelliptic systems, Calc. Var. Partial Differential Equations 5 (1997), 323-343.

${ }^{1}$ Department of Mathematics and Taida Institute for Mathematical Sciences (Tims), National Taiwan University, Taipei 10617, Taiwan, R.O.C.

E-mail address: scchang@math.ntu.edu.tw

2 School of Mathematics, Fudan University, Shanghai, 200433, P.R. China

E-mail address: yxdong@fudan.edu.cn

${ }^{3}$ College of Mathematics and Information Science, Xinyang Normal University, Xinyang,464000, Henan, P.R. China

E-mail address: yingbohan@163.com 